\title{
Delay-Dependent Synchronization for Complex Dynamical Networks with Interval Time-Varying and Switched Coupling Delays
}

\author{
T. Botmart ${ }^{1,2}$ and P. Niamsup ${ }^{2,3,4}$ \\ ${ }^{1}$ Department of Mathematics, Faculty of Science, Srinakharinwirot University, Bangkok 10110, Thailand \\ ${ }^{2}$ Center of Excellence in Mathematics CHE, Si Ayutthaya Road, Bangkok 10400, Thailand \\ ${ }^{3}$ Department of Mathematics, Faculty of Science, Chiang Mai University, Chiang Mai 50200, Thailand \\ ${ }^{4}$ Materials Science Research Center, Faculty of Science, Chiang Mai University, Chiang Mai 50200, Thailand \\ Correspondence should be addressed to P. Niamsup; piyapong.n@cmu.ac.th
}

Received 29 December 2012; Accepted 31 January 2013

Academic Editor: Xinzhi Liu

Copyright (C) 2013 T. Botmart and P. Niamsup. This is an open access article distributed under the Creative Commons Attribution License, which permits unrestricted use, distribution, and reproduction in any medium, provided the original work is properly cited.

\begin{abstract}
We investigate the local exponential synchronization for complex dynamical networks with interval time-varying delays in the dynamical nodes and the switched coupling term simultaneously. The constraint on the derivative of the time-varying delay is not required which allows the time delay to be a fast time-varying function. By using common unitary matrix for different subnetworks, the problem of synchronization is transformed into the stability analysis of some linear switched delay systems. Then, when subnetworks are synchronizable and nonsynchronizable, a delay-dependent sufficient condition is derived and formulated in the form of linear matrix inequalities (LMIs) by average dwell time approach and piecewise Lyapunov-Krasovskii functionals which are constructed based on the descriptor model of the system and the method of decomposition. The new stability condition is less conservative and is more general than some existing results. A numerical example is also given to illustrate the effectiveness of the proposed method.
\end{abstract}

\section{Introduction}

Complex dynamical network, as an interesting subject, has been thoroughly investigated for decades. These networks show very complicated behavior and can be used to model and explain many complex systems in nature such as computer networks [1], the World Wide Web [2], food webs [3], cellular and metabolic networks [4], social networks [5], and electrical power grids [6]. In general, a complex network is a large set of interconnected nodes, in which a node is a fundamental unit with specific contents. As an implicit assumption, these networks are described by the mathematical term graph. In such graphs, each vertex represents an individual element in the system, while edges represent the relationship between them. Two nodes are joined by an edge if and only if they interact.
In the last decade, the synchronization of complex dynamical networks has attracted much attention by several researchers in this field [7-12]. Synchronization of complex dynamical networks is one of the most important dynamical mechanisms for creating order in complex dynamical networks. Wang and Chen introduced a uniform dynamical network model and also investigated its synchronization [10, $11,13]$. They have shown that the synchronizability of a scalefree dynamical network is robust against random removal of nodes and yet is fragile to specific removal of the most highly connected nodes [11]. Li and Chen [7] considered the synchronization of complex dynamical network models with coupling delays (continuous and discrete-time) and derived delay-independent and delay-dependent synchronization conditions. By utilizing Lyapunov functional method, Yue and $\mathrm{Li}$ [12] studied the synchronization of continuous 
and discrete complex dynamical networks with interval time-varying delays in the dynamical nodes and the coupling term simultaneously in which delay-dependent synchronization conditions are derived in the form of linear matrix inequalities (LMIs). In [8], Li et al. studied the synchronization problem of general complex dynamical networks with time-varying delays in the network couplings and in the dynamical nodes, respectively. However, the time-varying delays are required to be differentiable.

It is well known that the existence of time delay in a system may cause instability and oscillations. Examples of time-delay systems are chemical engineering systems, biological modeling, electrical networks, physical networks, and many others, [14-20]. The stability criteria for systems with time delays can be classified into two categories: delayindependent and delay-dependent. Delay-independent criteria do not employ any information on the size of the delay, while delay-dependent criteria make use of such information at different levels. Delay-dependent stability conditions are generally less conservative than the delayindependent ones especially when the delay is small [20]. Recently, delay-dependent stability for interval time-varying delay was investigated in $[12,15-17,19]$. Interval time-varying delay is a time delay that varies in an interval in which the lower bound is not restricted to be 0. Jiang and Han [17] considered the problem of robust $H \infty$ control for uncertain linear systems with interval time-varying delay based on the Lyapunov functional approach in which restriction on the differentiability of the interval time-varying delay was removed. Shao [19] presented a new delay-dependent stability criterion for linear systems with interval time-varying delay without introducing any free-weighting matrices. In order to reduce further the conservatism introduced by descriptor model transformation and bounding techniques, a freeweighting matrices method is proposed in [15, 21-24]. An advantage of this transformation is to transform the original system to an equivalent descriptor representation without introducing additional dynamics in the sense defined in [21]. In [22], based on the descriptor model transformation and decomposition technique of coefficient matrix, some new robust stability criteria are derived. In [12], the synchronization problem has been investigated for continuous/discrete complex dynamical networks with interval timevarying delays. Based on piecewise analysis method and the Lyapunov functional method, some new delay-dependent synchronization criteria are derived in the form of LMIs by introducing free-weighting matrices. It will be pointed out later that some existing results require more free-weighting matrix variables than our result.

Switched systems are special class of hybrid systems which consist of a family of continuous-time or discretetime subsystems and a switching law that orchestrates the switching between them. Such systems have drawn considerable attention in control and computer communities in the last decade [25-31]. In [32], some stability properties of switched linear systems consisting of both stable and unstable subsystems have been derived by using an average dwell time approach and piecewise Lyapunov functions. It was shown that when the average dwell time is sufficiently large and the total activation time of the unstable subsystems is relatively small compared with that of stable subsystems, then global exponential stability is guaranteed. Liu and Zhao [9] investigated synchronization of complex delayed networks with switching topology via switched system stability theory. In [9], when all subnetworks are synchronizable, a delaydependent synchronization condition is given in terms of LMIs which guarantees the solvability of the synchronization problem under an average dwell time scheme. Liu et al. [33] studied the local and global exponential synchronization of a complex dynamical network with switching topology and time-varying coupling delays. However, the time-varying delays in the dynamical nodes were not considered.

In this paper, we will investigate the local exponential synchronization of complex dynamical networks with interval time-varying delays in both dynamical nodes and in switched coupling terms simultaneously. The restriction on the differentiability of interval time-varying delays is removed, which means that a fast time-varying delay is allowed. We use a common unitary matrix for different subnetworks to transform the synchronization problem of the network into the stability analysis of $N-1$ pieces of linear switched systems. Then by using the average dwell time approach, the descriptor model transformation, and the decomposition technique of coefficient matrix, a new class of piecewise Lyapunov-Krasovskii functionals are constructed in order to get improved delay-dependent synchronization criteria which are derived in the form of LMIs. Finally, numerical examples are given to demonstrate that the derived conditions are less conservative than some existing results given in the literature.

The rest of the paper is organized as follows. In Section 2, a complex dynamical network model with interval timevarying and switched coupling delays as well as some useful lemmas is given. In Section 3, common unitary matrix and descriptor system transformation strategy are introduced to reformulate the network model. Then delay-dependent synchronization criteria are presented based on this novel model. Numerical examples that illustrated the obtained results are given in Section 4. The paper ends with conclusions in Section 5 and cited references.

\section{Network Model and Mathematic Preliminaries}

The following notation will be used in this paper: $R^{n}$ denotes the $n$-dimensional Euclidean space; $\lambda_{\text {min }}(\cdot)$ and $\lambda_{\max }(\cdot)$ denote the minimum and maximum eigenvalue of a real symmetric matrix, respectively; $\|\cdot\|$ denotes the Euclidean vector norm of $x ; A^{T}$ denotes the transpose of the vector/matrix $A$; $A$ is symmetric if $A=A^{T}$; $I$ denotes the identity matrix; $\|\cdot\|_{\mathrm{cl}}=\sup _{-h_{M} \leq \theta \leq 0}\{\|x(t+\theta)\|,\|\dot{x}(t+\theta)\|\}$. The notation $\operatorname{diag}\{\ldots\}$ stands for a block-diagonal matrix. 
Consider a complex dynamical switched network consisting of $N$ identical coupled nodes, with each node being an $n$-dimensional dynamical system as

$$
\begin{aligned}
& \dot{x}_{i}(t)= f\left(x_{i}(t), x_{i}(t-h(t))\right) \\
&+c_{\sigma(t)} \sum_{j=1}^{N} g_{i j}^{\sigma(t)} A_{\sigma(t)} x_{j}(t-h(t)), \\
& x(t)=\phi(t), \quad t \in\left[t_{0}-h_{M}, t_{0}\right], h_{M} \geq 0,
\end{aligned}
$$

where $x_{i}(t)=\left(x_{i 1}(t), x_{i 2}(t), \ldots, x_{i n}(t)\right)^{T} \in R^{n}$ is the state vector of $i$ th node, for all $i \in\{1,2, \ldots, N\}$ are the state of node $i$ th; $f(\cdot) \in R^{n}$ is the continuously differentiable function; $\sigma(t):[0,+\infty) \rightarrow M=\{1,2, \ldots, m\}$ is piecewise constant and left continuous and is called a switching signal; the constant $c_{\sigma(t)}>0$ is the coupling strength; $A_{\sigma(t)}=\left(a_{i j}\right)_{n \times n}^{\sigma(t)} \in$ $R^{n \times n}$ is a constant inner-coupling matrix, if some pairs $(i, j)$, $1 \leq i, j \leq n$, with $a_{i j}^{\sigma(t)} \neq 0$ which means two coupled nodes are linked through their $i$ th and $j$ th state variables, otherwise $a_{i j}^{\sigma(t)}=0 ; G_{\sigma(t)}=\left(g_{i j}\right)_{N \times N}^{\sigma(t)} \in R^{N \times N}$ is the outer-coupling matrix of the network, in which $g_{i j}^{\sigma(t)}$ is defined as follows: if there is a connection between node $i$ and node $j(j \neq i)$, then $g_{i j}^{\sigma(t)}=g_{j i}^{\sigma(t)}=1$; otherwise, $g_{i j}^{\sigma(t)}=g_{j i}^{\sigma(t)}=0(j \neq i)$, and the diagonal elements of matrix $G$ are defined by

$$
g_{i i}^{\sigma(t)}=-\sum_{j=1, i \neq j}^{N} g_{i j}^{\sigma(t)}=-\sum_{j=1, i \neq j}^{N} g_{j i}^{\sigma(t)}, \quad i=1,2, \ldots, N .
$$

It is assumed that switched network (1) is connected in the sense that there are no isolated clusters; that is namely, $G_{\sigma(t)}$ is an irreducible matrix. The time delay $h(t)$ is a time-varying continuous function satisfying

$$
0 \leq h_{m} \leq h(t) \leq h_{M}
$$

The initial condition function $\phi(t)$ is a continuous vectorvalued function for $t \in\left[t_{0}-h_{M}, t_{0}\right]$.

Corresponding to the switching signal $\sigma(t)$, we have the switching sequence $\Sigma=\left\{x_{t_{0}} ;\left(i_{0}, t_{0}\right),\left(i_{1}, t_{1}\right), \ldots,\left(i_{k}, t_{k}\right), \ldots, \mid\right.$ $\left.i_{k} \in M\right\}$, and when $t \in\left[t_{k}, t_{k+1}\right)$, the $i_{k}$-th subnetwork is activated. Assume that there are no jumps in the state at the switching instants, and every network in (1) is connected; that is, $k \in M, G_{k}$ is irreducible.

Remark 1. For fixed $\sigma(t)$, the system model (1) turns into the delayed complex dynamical network proposed by Yue and $\mathrm{Li}$ $[12]$ as

$$
\begin{array}{r}
\dot{x}_{i}(t)=f\left(x_{i}(t), x_{i}(t-h(t))\right)+c \sum_{j=1}^{N} g_{i j} A x_{j}(t-h(t)), \\
i=1,2, \ldots, N .
\end{array}
$$

Therefore, (1) is a general complex networks model, with (4) as a special case.
Definition 2 (see [26]). For any $T_{2}>T_{1} \geq 0$, let $N_{\sigma(t)}\left(T_{1}, T_{2}\right)$ denote the number of switching of $\sigma(t)$ over $\left(T_{1}, T_{2}\right)$. If $N_{\sigma(t)}\left(T_{1}, T_{2}\right) \leq N_{0}+\left(\left(T_{2}-T_{1}\right) / T_{a}\right)$ holds for $T_{a}>0, N_{0} \geq 0$, then $T_{a}$ is called average dwell time and $N_{0}$ is called a chatter bound.

Definition 3 (see [12]). The delayed dynamical switched network (1) is said to achieve asymptotic synchronization if

$$
x_{1}(t)=x_{2}(t)=\cdots=s(t) \quad \text { as } t \longrightarrow \infty,
$$

where $s(t)$ is a solution of an isolated node satisfying

$$
\dot{s}(t)=f(s(t), s(t-h(t))) .
$$

Definition 4 (see [34]). If the matrix $A \in M_{n \times n}$ is similar to a diagonal matrix, then $A$ is said to be diagonalizable.

In this paper, we assume that $s(t)$ is an orbitally stable solution of the above system. Clearly, the stability of the synchronized states (5) of network (1) is determined by the dynamics of the isolated node, the coupling strength $c_{\sigma(t)}$, the inner-coupling matrix $A_{\sigma(t)}$, the outer-coupling matrix $G_{\sigma(t)}$, and the fast time-varying delays $h(t)$.

The following lemmas will be used in the proof of the main result.

Lemma 5 (see [34]). Let $G$ be a family of diagonalizable matrices. Then $G$ is a commuting family (under multiplication) if and only if it is a simultaneously diagonalizable family.

Lemma 6 (see [14]). For any constant symmetric matrix $M \in$ $R^{n \times n}, M=M^{T}>0,0 \leq h_{m} \leq h(t) \leq h_{M}, t \geq 0$, and any differentiable vector function $x(t) \in R^{n}$, we have

$$
\begin{gathered}
\text { (a) }\left[\int_{t-h_{m}}^{t} \dot{x}(s) d s\right]^{T} M\left[\int_{t-h_{m}}^{t} \dot{x}(s) d s\right] \\
\leq h_{m} \int_{t-h_{m}}^{t} \dot{x}^{T}(s) M \dot{x}(s) d s, \\
\text { (b) }\left[\int_{t-h(t)}^{t-h_{m}} \dot{x}(s) d s\right]^{T} M\left[\int_{t-h(t)}^{t-h_{m}} \dot{x}(s) d s\right] \\
\leq\left(h(t)-h_{m}\right) \int_{t-h(t)}^{t-h_{m}} \dot{x}^{T}(s) M \dot{x}(s) d s \\
\leq\left(h_{M}-h_{m}\right) \int_{t-h(t)}^{t-h_{m}} \dot{x}^{T}(s) M \dot{x}(s) d s .
\end{gathered}
$$

\section{Synchronization of Switched Network}

In this section, we will obtain some delay-dependent synchronization criteria for general complex dynamical network with interval time-varying delay and switched coupling delays (1) by strict LMI approaches. Let $\widetilde{A}_{i}=J(t), \widetilde{B}_{i \sigma(t)}=$ $J_{h}(t-h(t))+c_{\sigma(t)} \lambda_{i \sigma(t)} A_{\sigma(t)}, J(t)=f^{\prime}(s(t)$, and $s(t-$ $h(t))) \in R^{n \times n}$ is the Jacobian of $f(x(t), x(t-h(t)))$ at $s(t)$ with the derivative of $f(x(t), x(t-h(t)))$ with respect to $x(t)$. 
$J_{h}(t-h(t))=f^{\prime}(s(t), s(t-h(t))) \in R^{n \times n}$ is the Jacobian of $f(x(t), x(t-h(t)))$ at $s(t-h(t))$ with the derivative of $f(x(t), x(t-h(t)))$ with respect to $x(t-h(t))$.

Lemma 7. Consider the switched coupling delays dynamical network described by (1). Let $0=\lambda_{1 \sigma(t)}>\lambda_{2 \sigma(t)} \geq \lambda_{3 \sigma(t)} \geq$ $\cdots \geq \lambda_{N \sigma(t)}$ be the eigenvalues of the outer-coupling matrix $G_{\sigma(t)}$. If the n-dimensional linear switched system

$$
\dot{z}_{i}(t)=\widetilde{A}_{i} z_{i}(t)+\widetilde{B}_{i \sigma(t)} z_{i}(t-h(t)), \quad i=2,3, \ldots, N
$$

is asymptotically (or exponentially) stable, then the synchronized state (5) is asymptotically (or exponentially) stable.

Proof. To investigate the stability of the synchronized states (5), set

$$
e_{i}(t)=x_{i}(t)-s(t), \quad i=1,2, \ldots, N
$$

Substituting (9) into (1), for $1 \leq i \leq N$, we have

$$
\begin{aligned}
\dot{e}_{i}(t)= & \dot{x}_{i}(t)-\dot{s}(t) \\
= & f\left(x_{i}(t), x_{i}(t-h(t))\right)-f(s(t), s(t-h(t))) \\
& +c_{\sigma(t)} \sum_{j=1}^{N} g_{i j}^{\sigma(t)} A_{\sigma(t)} e_{j}(t-h(t)) .
\end{aligned}
$$

Since $f(\cdot)$ is continuous differentiable, it is easy to show that the origin of the nonlinear system (1) is an asymptotically stable equilibrium point if it is an asymptotically stable equilibrium point of the following linear switched timevarying delays systems:

$$
\begin{aligned}
\dot{e}_{i}(t)= & J(t) e_{i}(t)+J_{h}(t) e_{i}(t-h(t)) \\
& +c_{\sigma(t)} \sum_{j=1}^{N} g_{i j}^{\sigma(t)} A_{\sigma(t)} e_{j}(t-h(t)) \\
= & J(t) e_{i}(t)+J_{h}(t) e_{i}(t-h(t)) \\
& +c_{\sigma(t)} A_{\sigma(t)}\left(e_{1}(t-h(t)), \ldots, e_{N}(t-h(t))\right) \\
& \times\left(g_{i 1}^{\sigma(t)}, \ldots, g_{i N}^{\sigma(t)}\right)^{T},
\end{aligned}
$$

where $J(t)=f^{\prime}(s(t), s(t-h(t))) \in R^{n \times n}$ is the Jacobian of $f(x(t), x(t-h(t)))$ at $s(t)$ and $J_{h}(t-h(t))=f^{\prime}(s(t), s(t-$ $h(t))) \in R^{n \times n}$ is the Jacobian of $f(x(t), x(t-h(t)))$ at $s(t-h(t))$.

Letting $e(t)=\left(e_{1}(t), \ldots, e_{N}(t)\right) \in R^{n \times N}$ and $e(t-h(t))=$ $\left(e_{1}(t-h(t)), \ldots, e_{N}(t-h(t))\right) \in R^{n \times N}$, we have

$$
\begin{aligned}
\dot{e}(t)= & J(t) e(t)+J_{h}(t-h(t)) e(t-h(t)) \\
& +c_{\sigma(t)} A_{\sigma(t)} e(t-h(t)) G_{\sigma(t)}^{T} .
\end{aligned}
$$

Obviously, $G_{k}$ is diagonalizable for fixed $\sigma(t)=k \in M$. If $G_{k}$, $G_{l}$, for all $k$, and $l \in M$ commute pairwise; that is, $G_{k} G_{l}=$ $G_{l} G_{k}$, then based on Lemma 5 , one can get a common unitary matrix $U \in R^{N \times N}$ with $u_{i} \in R^{N}$ such that

$$
U^{T} G_{k} U=\Gamma_{k},
$$

where $U^{T} U=I, \Gamma_{k}=\operatorname{diag}\left\{\lambda_{1 k}, \ldots, \lambda_{N k}\right\}, \lambda_{i k}, i=1,2, \ldots, N$ are eigenvalues of $G_{k}$. In addition, with (2) and the irreducible feature of $G_{k}$, we may choose $u_{1}=(1 / \sqrt{N})(1,1, \ldots, 1)^{T}$ such that $\lambda_{1 k}=0$, for all $k \in M$.

From (12), by using the nonsingular transform $e(t) U=$ $z(t)=\left(z_{1}(t), \ldots, z_{N}(t)\right) \in R^{N \times N}$, we have the following matrix equation:

$$
\begin{aligned}
\dot{z}(t)= & J(t) z(t)+J_{h}(t-h(t)) z(t-h(t)) \\
& +c_{\sigma(t)} A_{\sigma(t)} z(t-h(t)) \Gamma_{\sigma(t)},
\end{aligned}
$$

namely,

$$
\dot{z}_{i}(t)=\widetilde{A}_{i} z_{i}(t)+\widetilde{B}_{i \sigma(t)} z_{i}(t-h(t)), \quad i=1,2, \ldots, N
$$

where $\widetilde{A}_{i}=J(t)$ and $\widetilde{B}_{i \sigma(t)}=J_{h}(t-h(t))+c_{\sigma(t)} \lambda_{i \sigma(t)} A_{\sigma(t)}$. Thus, we have transformed the stability problem of the synchronized state (5) to the stability problem of the $N$ pieces of $n$-dimensional linear switched time-varying delays differential equations (15). Note that $\lambda_{1 k}=0$ corresponding to the synchronization of the system states (5), where the state $s(t)$ is an orbitally stable solution of the isolated node as assumed above in (5). If the following $N-1$ pieces of $n$ dimensional linear switched time-varying delays systems

$$
\dot{z}_{i}(t)=\widetilde{A}_{i} z_{i}(t)+\widetilde{B}_{i \sigma(t)} z_{i}(t-h(t)), \quad i=2,3, \ldots, N,
$$

are asymptotically (or exponentially) stable, then $e(t)$ will tend to the origin asymptotically (or exponentially), which is equivalent to the fact that the synchronized states (5) are asymptotically (or exponentially) stable. This completes the proof.

Next, we consider the nonswitched system

$$
\dot{z}_{i}(t)=\widetilde{A}_{i} z_{i}(t)+\widetilde{B}_{i} z_{i}(t-h(t)), \quad i=2,3, \ldots, N .
$$

We rewrite system (17) in the following equivalent descriptor system form:

$$
\begin{gathered}
\dot{z}_{i}(t)=y_{i}(t) \\
y_{i}(t)=\widetilde{A}_{i} z_{i}(t)+\widetilde{B}_{i} z_{i}(t-h(t)), \quad i=2,3, \ldots, N .
\end{gathered}
$$

To derive delay-dependent stability conditions, which include the information of the time-varying delay $h(t)$, one usually uses the fact

$$
\begin{aligned}
z(t-h(t)) & =z(t)-\int_{t-h(t)}^{t} \dot{z}(s) d s \\
& =z(t)-\int_{t-h(t)}^{t} y(s) d s
\end{aligned}
$$

to transform the original system to a system with distributed delays. In order to improve the bound of time-varying delay $h(t)$, let us decompose the matrix $\widetilde{B}_{i}=\widetilde{B}_{i 1}+\widetilde{B}_{i 2}$, where $\widetilde{B}_{i 1}$ are $\widetilde{B}_{i 2}$ are appropriate constant matrices to be determined later. Then the original system (17) can be represented in the 
form of a descriptor system with time-varying and distributed delays as

$$
\begin{gathered}
\dot{z}_{i}(t)=y_{i}(t) \\
0=-y_{i}(t)+\left(\widetilde{A}_{i}+\widetilde{B}_{i 1}\right) z_{i}(t)+\widetilde{B}_{i 2} z_{i}(t-h(t)) \\
-\widetilde{B}_{i 1} \int_{t-h(t)}^{t} y_{i}(s) d s, \quad i=2,3, \ldots, N .
\end{gathered}
$$

Let $\delta=h_{M}-h_{m}$. Let $V_{i}(t)$ be defined by

$$
V_{i}(t)=V_{i 1}(t)+V_{i 2}(t)+V_{i 3}(t)+V_{i 4}(t)+V_{i 5}(t)+V_{i 6}(t),
$$

where

$$
\begin{aligned}
V_{i 1}(t) & =\xi_{i}^{T}(t) E P_{i} \xi_{i}(t)=z_{i}^{T}(t) P_{i 1} z_{i}(t), \\
V_{i 2}(t) & =\int_{t-h_{m}}^{t} z_{i}^{T}(s) Q_{i} e^{\alpha(s-t)} z_{i}(s) d s, \\
V_{i 3}(t) & =\int_{t-h_{M}}^{t} z_{i}^{T}(s) R_{i} e^{\alpha(s-t)} z_{i}(s) d s, \\
V_{i 4}(t) & =h_{m} \int_{-h_{m}}^{0} \int_{t+\theta}^{t} \dot{z}_{i}^{T}(s) U_{i} e^{\alpha(s-t)} \dot{z}_{i}(s) d s d \theta, \\
V_{i 5}(t) & =h_{M} \int_{-h_{M}}^{0} \int_{t+\theta}^{t} y_{i}^{T}(s) S_{i} e^{\alpha(s-t)} y_{i}(s) d s d \theta, \\
V_{i 6}(t) & =\delta \int_{-h_{M}}^{-h_{m}} \int_{t+\theta}^{t} \dot{z}_{i}^{T}(s) W_{i} e^{\alpha(s-t)} \dot{z}_{i}(s) d s d \theta, \\
\xi_{i}(t) & =\left[\begin{array}{lll}
z_{i}^{T}(t) & y_{i}^{T}(t) \int_{t-h(t)}^{t} y_{i}^{T}(s) d s
\end{array}\right]^{T}, \\
E & =\left[\begin{array}{lll}
I & 0 & 0 \\
0 & 0 & 0
\end{array}\right]^{T}, \quad P_{i}=\left[\begin{array}{lll}
P_{i 1} & 0 & 0 \\
P_{i 2} & P_{i 3} & P_{i 4}
\end{array}\right] .
\end{aligned}
$$

For given $\alpha>0$, the following lemma provides an estimation of $V_{i}(t)$.

Lemma 8. Assume that the time-varying delay $h(t)$ satisfies $0 \leq h_{m} \leq h(t) \leq h_{M}$ and let $\alpha>0$. If there exist symmetric positive definite matrices $P_{i 1}>0, Q_{i}>0, R_{i}>0, S_{i}>0$, $U_{i}>0$, and $W_{i}>0$ and appropriately dimensioned matrices $P_{i 2}, P_{i 3}$, and $P_{i 4}$ for $i=2,3, \ldots, N$, such that the following LMIs hold:

$$
\begin{aligned}
\Sigma_{i 1}= & \Sigma_{i}-\left[\begin{array}{llllll}
0 & 0 & I & -I & 0 & 0
\end{array}\right]^{T} \\
& \times e^{-\alpha \delta} W_{i}\left[\begin{array}{llllll}
0 & 0 & I & -I & 0 & 0
\end{array}\right]<0, \\
\Sigma_{i 2}= & \Sigma_{i}-\left[\begin{array}{llllll}
0 & 0 & 0 & I & -I & 0
\end{array}\right]^{T} \\
& \times e^{-\alpha \delta} W_{i}\left[\begin{array}{llllll}
0 & 0 & 0 & I & -I & 0
\end{array}\right]<0,
\end{aligned}
$$

$i=2, \ldots, N$, where

$$
\begin{aligned}
\Sigma_{i}= & {\left[\begin{array}{cccccc}
\Sigma_{i 11} & \Sigma_{i 12} & \Sigma_{i 13} & \Sigma_{i 14} & 0 & \Sigma_{i 16} \\
* & \Sigma_{i 22} & 0 & \Sigma_{i 24} & 0 & \Sigma_{i 26} \\
* & * & \Sigma_{i 33} & \Sigma_{i 34} & 0 & 0 \\
* & * & * & \Sigma_{i 44} & \Sigma_{i 45} & \Sigma_{i 46} \\
* & * & * & * & \Sigma_{i 55} & 0 \\
* & * & * & * & * & \Sigma_{i 66}
\end{array}\right], } \\
\Sigma_{i 11}= & P_{i 2}^{T}\left(\widetilde{A}_{i}+\widetilde{B}_{i 1}\right)+\left(\widetilde{A}_{i}+\widetilde{B}_{i 1}\right)^{T} P_{i 2}+Q_{i} \\
& +R_{i}-e^{-\alpha h_{m}} U_{i}+\alpha P_{i 1},
\end{aligned}
$$

$$
\Sigma_{i 12}=P_{i 1}^{T}-P_{i 2}^{T}+\left(\widetilde{A}_{i}+\widetilde{B}_{i 1}\right)^{T} P_{i 3} \text {, }
$$

$$
\begin{aligned}
& \Sigma_{i 13}=e^{-\alpha h_{M}} U_{i}, \\
& \Sigma_{i 14}=P_{i 2}^{T} \widetilde{B}_{i 2}, \\
& \Sigma_{i 16}=-P_{i 2}^{T} \widetilde{B}_{i 1}-\left(\widetilde{A}_{i}+\widetilde{B}_{i 1}\right)^{T} P_{i 4}, \\
& \Sigma_{i 22}=h_{m}^{2} U_{i}+h_{M}^{2} S_{i}+\delta^{2} W_{i}-P_{i 3}^{T}-P_{i 3}, \\
& \Sigma_{i 24}=P_{i 3}^{T} \widetilde{B}_{i 2}, \\
& \Sigma_{i 26}=-P_{i 3}^{T} \widetilde{B}_{i 1}-P_{i 4}, \\
& \Sigma_{i 33}=-e^{-\alpha h_{m}} Q_{i}-e^{-\alpha h_{m}} U_{i}-W_{i}, \\
& \Sigma_{i 34}=e^{-\alpha \delta} W_{i}, \\
& \Sigma_{i 44}=-2 e^{-\alpha \delta} W_{i}, \\
& \Sigma_{i 45}=e^{-\alpha \delta} W_{i}, \\
& \Sigma_{i 46}=\widetilde{B}_{i 2} P_{i 4}^{T}, \\
& \sum_{i 55}=-e^{-\alpha h_{M}} R_{i}-e^{-\alpha \delta} W_{i}, \\
& \sum_{i 66}=-e^{-\alpha h_{M}} S_{i}-P_{i 4}^{T} \widetilde{B}_{i 1}-\widetilde{B}_{i 1}^{T} P_{i 4} .
\end{aligned}
$$

Then, along the trajectory of system (17), we have

$$
V_{i}(t) \leq e^{-\alpha\left(t-t_{0}\right)} V_{i}\left(t_{0}\right), \quad t \geq t_{0} \geq 0 .
$$

Proof. By taking the derivative of Lyapunov-Krasovskii functional candidate (22) along the trajectory of the system (21), we obtain

$\dot{V}_{i}(t)=\dot{V}_{i 1}(t)+\dot{V}_{i 2}(t)+\dot{V}_{i 3}(t)+\dot{V}_{i 4}(t)+\dot{V}_{i 5}(t)+\dot{V}_{i 6}(t)$, 
where

$$
\begin{aligned}
& \dot{V}_{i 1}(t)= 2 z_{i}^{T}(t) P_{i 1} \dot{z}_{i}(t) \\
&= 2 \xi_{i}^{T}(t) P_{i}^{T}\left[\begin{array}{c}
\dot{z}_{i}(t) \\
0
\end{array}\right], \\
& \dot{V}_{i 2}(t)=-\alpha V_{i 2}(t)+z_{i}^{T}(t) Q_{i} z_{i}(t) \\
&-z_{i}^{T}\left(t-h_{m}\right) Q_{i} e^{-\alpha h_{m}} z_{i}\left(t-h_{m}\right), \\
& \dot{V}_{i 3}(t)=-\alpha V_{i 3}(t)+z_{i}^{T}(t) R_{i} z_{i}(t) \\
&-z_{i}^{T}\left(t-h_{M}\right) R_{i} e^{-\alpha h_{M}} z_{i}\left(t-h_{M}\right), \\
& \dot{V}_{i 4}(t) \leq-\alpha V_{i 4}(t)+y_{i}^{T}(t) h_{m}^{2} U_{i} y_{i}(t) \\
&-h_{m} e^{-\alpha h_{m}} \int_{t-h_{m}}^{t} \dot{z}_{i}^{T}(s) U_{i} \dot{z}_{i}(s) d s, \\
& \dot{V}_{i 6}(t) \leq-\alpha V_{i 6}(t)+y_{i}^{T}(t) \delta^{2} W_{i} y_{i}(t) \\
& \dot{V}_{i 5}(t) \leq-\alpha V_{i 5}(t)+y_{i}^{T}(t) h_{M}^{2} S_{i} y_{i}(t) \\
&-h_{M} e^{-\alpha h_{M}} \int_{t-h_{m}}^{t} \dot{z}_{i}^{T}(s) W_{i} \dot{z}_{i}(s) d s . \\
& y_{i}^{T}(s) S_{i} y_{i}(s) d s,
\end{aligned}
$$

Using Lemma 6, we obtain

$$
\begin{aligned}
-h_{m} & \int_{t-h_{m}}^{t} \dot{z}_{i}^{T}(s) U_{i} \dot{z}_{i}(s) d s \\
\leq & -\left[\int_{t-h_{m}}^{t} \dot{z}_{i}^{T}(s) d s\right]^{T} U_{i}\left[\int_{t-h_{m}}^{t} \dot{z}_{i}^{T}(s) d s\right] \\
= & -\left[z_{i}(t)-z_{i}\left(t-h_{m}\right)\right]^{T} U_{i}\left[z_{i}(t)-z_{i}\left(t-h_{m}\right)\right] \\
= & -z_{i}^{T}(t) U_{i} z_{i}(t)+2 z_{i}^{T}(t) U_{i} z_{i}\left(t-h_{m}\right) \\
& -z_{i}^{T}\left(t-h_{m}\right) U_{i} z_{i}\left(t-h_{m}\right) \\
-h_{M} & \int_{t-h_{M}}^{t} y_{i}^{T}(s) S_{i} y_{i}(s) d s \\
\leq & -h(t) \int_{t-h(t)}^{t} y_{i}^{T}(s) S_{i} y_{i}(s) d s \\
\leq & -\left[\int_{t-h(t)}^{t} y_{i}^{T}(s) d s\right]^{T} S_{i}\left[\int_{t-h(t)}^{t} y_{i}^{T}(s) d s\right] .
\end{aligned}
$$

On the other hand, we have

$$
\begin{aligned}
-\delta \int_{t-h_{M}}^{t-h_{m}} \dot{z}_{i}^{T}(s) W_{i} \dot{z}_{i}(s) d s & \\
= & -\delta \int_{t-h(t)}^{t-h_{m}} \dot{z}_{i}^{T}(s) W_{i} \dot{z}_{i}(s) d s \\
& -\delta \int_{t-h_{M}}^{t-h(t)} \dot{z}_{i}^{T}(s) W_{i} \dot{z}_{i}(s) d s \\
= & -\left(h_{M}-h(t)\right) \int_{t-h(t)}^{t-h_{m}} \dot{z}_{i}^{T}(s) W_{i} \dot{z}_{i}(s) d s \\
& -\left(h(t)-h_{m}\right) \int_{t-h(t)}^{t-h_{m}} \dot{z}_{i}^{T}(s) W_{i} \dot{z}_{i}(s) d s \\
& -\left(h_{M}-h(t)\right) \int_{t-h_{M}}^{t-h(t)} \dot{z}_{i}^{T}(s) W_{i} \dot{z}_{i}(s) d s \\
& -\left(h(t)-h_{m}\right) \int_{t-h_{M}}^{t-h(t)} \dot{z}_{i}^{T}(s) W_{i} \dot{z}_{i}(s) d s .
\end{aligned}
$$

Let $\gamma=\left(h(t)-h_{m}\right) / \delta$. Then, we obtain

$$
\begin{aligned}
& -\left(h(t)-h_{m}\right) \int_{t-h_{M}}^{t-h(t)} \dot{z}_{i}^{T}(s) W_{i} \dot{z}_{i}(s) d s \\
& \quad=-\gamma \int_{t-h_{M}}^{t-h(t)} \delta \dot{z}_{i}^{T}(s) W_{i} \dot{z}_{i}(s) d s \\
& \quad \leq-\gamma \int_{t-h_{M}}^{t-h(t)}\left(h_{M}-h(t)\right) \dot{z}_{i}^{T}(s) W_{i} \dot{z}_{i}(s) d s, \\
& -\left(h_{M}-h(t)\right) \int_{t-h(t)}^{t-h_{m}} \dot{z}_{i}^{T}(s) W_{i} \dot{z}_{i}(s) d s \\
& \quad=-(1-\gamma) \int_{t-h(t)}^{t-h_{m}} \delta \dot{z}_{i}^{T}(s) W_{i} \dot{z}_{i}(s) d s \\
& \quad \leq-(1-\gamma) \int_{t-h(t)}^{t-h_{m}}\left(h(t)-h_{m}\right) \dot{z}_{i}^{T}(s) W_{i} \dot{z}_{i}(s) d s .
\end{aligned}
$$

From Lemma 6, we have

$$
\begin{aligned}
-\delta \int_{t-h_{M}}^{t-h_{m}} \dot{z}_{i}^{T}(s) W_{i} \dot{z}_{i}(s) d s \\
\leq-\left[z_{i}\left(t-h_{m}\right)-z_{i}(t-h(t))\right]^{T} \\
\times W_{i}\left[z_{i}\left(t-h_{m}\right)-z_{i}(t-h(t))\right] \\
-\left[z_{i}(t-h(t))-z_{i}\left(t-h_{M}\right)\right]^{T}
\end{aligned}
$$




$$
\begin{aligned}
& \times W_{i}\left[z_{i}(t-h(t))-z_{i}\left(t-h_{M}\right)\right] \\
& -\gamma\left[z_{i}(t-h(t))-z_{i}\left(t-h_{M}\right)\right]^{T} \\
& \times W_{i}\left[z_{i}(t-h(t))-z_{i}\left(t-h_{M}\right)\right] \\
& -(1-\gamma)\left[z_{i}\left(t-h_{m}\right)-z_{i}(t-h(t))\right]^{T} \\
& \times W_{i}\left[z_{i}\left(t-h_{m}\right)-z_{i}(t-h(t))\right] .
\end{aligned}
$$

Therefore, from (21) and (30)-(34), it follows that

$$
\begin{aligned}
\dot{V}_{i}(t)+\alpha V_{i}(t) \leq & \xi_{1}^{T}(t) \Sigma_{i} \xi_{1}(t) \\
& -\gamma\left[z_{i}(t-h(t))-z_{i}\left(t-h_{M}\right)\right]^{T} \\
& \times e^{-\alpha \delta} W_{i}\left[z_{i}(t-h(t))-z_{i}\left(t-h_{M}\right)\right] \\
& -(1-\gamma)\left[z_{i}\left(t-h_{m}\right)-z_{i}(t-h(t))\right]^{T} \\
& \times e^{-\alpha \delta} W_{i}\left[z_{i}\left(t-h_{m}\right)-z_{i}(t-h(t))\right] \\
= & \xi_{1}^{T}(t)\left[\gamma \Sigma_{i 1}+(1-\gamma) \Sigma_{i 2}\right] \xi_{1}(t),
\end{aligned}
$$

where $\Sigma_{i 1}, \Sigma_{i 2}$, and $\Sigma_{i}$ are defined in (24), (25), and (26), respectively, and

$$
\begin{gathered}
\xi_{1}(t)=\left[\begin{array}{llll}
z_{i}^{T}(t) & y_{i}^{T}(t) & z_{i}^{T}\left(t-h_{m}\right) & z_{i}^{T}(t-h(t)) \\
z_{i}^{T}\left(t-h_{M}\right) & \int_{t-h(t)}^{t} y_{i}^{T}(s) d s
\end{array}\right]^{T}
\end{gathered}
$$

Since $0 \leq \gamma \leq 1, \gamma \Sigma_{i 1}+(1-\gamma) \Sigma_{i 2}$ is a convex combination of $\Sigma_{i 1}$ and $\Sigma_{i 2}$. Therefore, $\gamma \Sigma_{i 1}+(1-\gamma) \Sigma_{i 1}<0$ is equivalent to $\Sigma_{i 1}<0$ and $\Sigma_{i 2}<0$. Thus, it follows from (24), (25), and (35) that

$$
\dot{V}_{i}(t)+\alpha V_{i}(t) \leq 0, \quad t \geq t_{0} \geq 0 .
$$

By integrating this inequality from $t_{0}$ to $t$, we obtain (27).

Remark 9. If $\alpha=0$ in (24) and (25), then it follows from Lemma 8 that the network system (17) is asymptotically stable.
Next, we define $V(t)$ as follows:

$$
V_{i}(t)=V_{i 1}(t)+V_{i 2}(t)+V_{i 3}(t)+V_{i 4}(t)+V_{i 5}(t)+V_{i 6}(t),
$$

where

$$
\begin{aligned}
& V_{i 1}(t)=\xi_{i}^{T}(t) E P_{i} \xi_{i}(t)=z_{i}^{T}(t) P_{i 1} z_{i}(t), \\
& V_{i 2}(t)=\int_{t-h_{m}}^{t} z_{i}^{T}(s) Q_{i} e^{\beta(t-s)} z_{i}(s) d s, \\
& V_{i 3}(t)=\int_{t-h_{M}}^{t} z_{i}^{T}(s) R_{i} e^{\beta(t-s)} z_{i}(s) d s, \\
& V_{i 4}(t)=h_{m} \int_{-h_{m}}^{0} \int_{t+\theta}^{t} \dot{z}_{i}^{T}(s) U_{i} e^{\beta(t-s)} \dot{z}_{i}(s) d s d \theta, \\
& V_{i 5}(t)=h_{M} \int_{-h_{M}}^{0} \int_{t+\theta}^{t} y_{i}^{T}(s) S_{i} e^{\beta(t-s)} y_{i}(s) d s d \theta, \\
& V_{i 6}(t)=\delta \int_{-h_{M}}^{-h_{m}} \int_{t+\theta}^{t} \dot{z}_{i}^{T}(s) W_{i} e^{\beta(t-s)} \dot{z}_{i}(s) d s d \theta, \\
& \xi_{i}(t)=\left[\begin{array}{lll}
z_{i}^{T}(t) & y_{i}^{T}(t) & \int_{t-h(t)}^{t} y_{i}^{T}(s) d s
\end{array}\right]^{T}, \\
& E=\left[\begin{array}{lll}
I & 0 & 0 \\
0 & 0 & 0
\end{array}\right]^{T}, \quad P_{i}=\left[\begin{array}{ccc}
P_{i 1} & 0 & 0 \\
P_{i 2} & P_{i 3} & P_{i 4}
\end{array}\right] .
\end{aligned}
$$

For given $\alpha>0$, the following lemma provides an estimation of $V_{i}(t)$.

Lemma 10. Assume that the time-varying delay $h(t)$ satisfies $0 \leq h_{m} \leq h(t) \leq h_{M}$ and let $\beta>0$. If there exist symmetric positive definite matrices $P_{i 1}>0, Q_{i}>0, R_{i}>0, S_{i}>0, U_{i}>0$, and $W_{i}>0$ and appropriately dimensioned matrices $P_{i 2}, P_{i 3}$, and $P_{i 4}$ for $i=2,3, \ldots, N$, such that the following symmetric LMIs hold:

$$
\begin{aligned}
\Sigma_{i 1}= & \Sigma_{i}-\left[\begin{array}{llllll}
0 & 0 & I & -I & 0 & 0
\end{array}\right]^{T} \\
& \times W_{i}\left[\begin{array}{llllll}
0 & 0 & I & -I & 0 & 0
\end{array}\right]<0, \\
\Sigma_{i 2}= & \Sigma_{i}-\left[\begin{array}{llllll}
0 & 0 & 0 & I & -I & 0
\end{array}\right]^{T} \\
& \times W_{i}\left[\begin{array}{llllll}
0 & 0 & 0 & I & -I & 0
\end{array}\right]<0,
\end{aligned}
$$


$i=2, \ldots, N$, where

$$
\begin{aligned}
& \Sigma_{i}=\left[\begin{array}{cccccc}
\Sigma_{i 11} & \Sigma_{i 12} & \Sigma_{i 13} & \Sigma_{i 14} & 0 & \Sigma_{i 16} \\
* & \Sigma_{i 22} & 0 & \Sigma_{i 24} & 0 & \Sigma_{i 26} \\
* & * & \Sigma_{i 33} & \Sigma_{i 34} & 0 & 0 \\
* & * & * & \Sigma_{i 44} & \sum_{i 45} & \Sigma_{i 46} \\
* & * & * & * & \sum_{i 55} & 0 \\
* & * & * & * & * & \Sigma_{i 66}
\end{array}\right] \\
& \Sigma_{i 11}=P_{i 2}^{T}\left(\widetilde{A}_{i}+\widetilde{B}_{i 1}\right)+\left(\widetilde{A}_{i}+\widetilde{B}_{i 1}\right)^{T} P_{i 2} \\
& +Q_{i}+R_{i}-U_{i}-\beta P_{i 1} \text {, } \\
& \Sigma_{i 12}=P_{i 1}^{T}-P_{i 2}^{T}+\left(\widetilde{A}_{i}+\widetilde{B}_{i 1}\right)^{T} P_{i 3}, \\
& \Sigma_{i 13}=U_{i}, \\
& \Sigma_{i 14}=P_{i 2}^{T} \widetilde{B}_{i 2} \text {, } \\
& \Sigma_{i 16}=-P_{i 2}^{T} \widetilde{B}_{i 1}-\left(\widetilde{A}_{i}+\widetilde{B}_{i 1}\right)^{T} P_{i 4}, \\
& \Sigma_{i 22}=h_{m}^{2} U_{i}+h_{M}^{2} S_{i}+\delta^{2} W_{i}-P_{i 3}^{T}-P_{i 3}, \\
& \Sigma_{i 24}=P_{i 3}^{T} \widetilde{B}_{i 2} \text {, } \\
& \Sigma_{i 26}=-P_{i 3}^{T} \widetilde{B}_{i 1}-P_{i 4}, \\
& \Sigma_{i 33}=-Q_{i}-U_{i}-W_{i}, \\
& \Sigma_{i 34}=W_{i} \text {, } \\
& \Sigma_{i 44}=-2 W_{i}, \\
& \Sigma_{i 45}=W_{i}, \\
& \Sigma_{i 46}=\widetilde{B}_{i 2} P_{i 4}^{T} \text {, } \\
& \sum_{i 55}=-R_{i}-W_{i}, \\
& \Sigma_{i 66}=-S_{i}-P_{i 4}^{T} \widetilde{B}_{i 1}-\widetilde{B}_{i 1}^{T} P_{i 4} .
\end{aligned}
$$

Then, along the trajectory of system (17), we have

$$
V_{i}(t) \leq e^{\beta\left(t-t_{0}\right)} V_{i}\left(t_{0}\right), \quad t \geq t_{0} \geq 0
$$

Proof. With the same argument as in Lemma 8, by taking the derivative of Lyapunov-Krasovskii functional candidate (38) along the trajectory of the system (21), we obtain

$$
\dot{V}_{i}(t)-\beta V_{i}(t) \leq 0, \quad t \geq t_{0} \geq 0
$$

Integrating this inequality from $t_{0}$ to $t$, we get (42).

We are now ready to derive some new sufficient conditions for exponential synchronization of the switched network

$$
\dot{z}_{i}(t)=\widetilde{A}_{i} z_{i}(t)+\widetilde{B}_{i \sigma(t)} z_{i}(t-h(t)), \quad i=2,3, \ldots, N
$$

We rewrite system (44) in the following equivalent descriptor system form:

$$
\begin{gathered}
\dot{z}_{i}(t)=y_{i}(t), \\
0=-y_{i}(t)+\left(\widetilde{A}_{i}+\widetilde{B}_{i \sigma(t) 1}\right) z_{i}(t) \\
+\widetilde{B}_{i \sigma(t) 2} z_{i}(t-h(t))-\widetilde{B}_{i \sigma(t) 1} \int_{t-h(t)}^{t} y_{i}(s) d s .
\end{gathered}
$$

We consider the case when synchronizable and nonsynchronizable subnetworks coexist. Without loss of generality, we suppose that each subnetwork $k \in S_{s}=\{1,2, \ldots, r\}$ is synchronizable, where $1 \leq r<m$, and each subnetwork $l \in S_{n}=\{r+1, r+2, \ldots, m\}$ is non-synchronizable.

For each subnetwork of the system (45) which satisfies Lemma 8, the Lyapunov-Krasovskii functional candidate can be chosen as

$$
\begin{aligned}
V_{i k}(t)= & z_{i}^{T}(t) E P_{i k 1} z_{i}(t)+\int_{t-h_{m}}^{t} z_{i}^{T}(s) Q_{i k} e^{\alpha(s-t)} z_{i}(s) d s \\
& +\int_{t-h_{M}}^{t} z_{i}^{T}(s) R_{i k} e^{\alpha(s-t)} z_{i}(s) d s \\
& +h_{m} \int_{-h_{m}}^{0} \int_{t+\theta}^{t} \dot{z}_{i}^{T}(s) U_{i k} e^{\alpha(s-t)} \dot{z}_{i}(s) d s d \theta \\
& +h_{M} \int_{-h_{M}}^{0} \int_{t+\theta}^{t} y_{i}^{T}(s) S_{i k} e^{\alpha(s-t)} y_{i}(s) d s d \theta \\
& +\delta \int_{-h_{M}}^{-h_{m}} \int_{t+\theta}^{t} \dot{z}_{i}^{T}(s) W_{i k} e^{\alpha(s-t)} \dot{z}_{i}(s) d s d \theta,
\end{aligned}
$$

where $i=2,3, \ldots, N, k \in S_{s}, P_{i k 1}, Q_{i k}, R_{i k}, U_{i k}, S_{i k}$, and $W_{i k}$ are positive definite matrices and

$$
\begin{aligned}
\xi_{i}(t) & =\left[\begin{array}{lll}
z_{i}^{T}(t) & y_{i}^{T}(t) & \int_{t-h(t)}^{t} y_{i}^{T}(s) d s
\end{array}\right]^{T}, \\
E & =\left[\begin{array}{lll}
I & 0 & 0 \\
0 & 0 & 0
\end{array}\right]^{T}, \quad P_{i k}=\left[\begin{array}{ccc}
P_{i k 1} & 0 & 0 \\
P_{i k 2} & P_{i k 3} & P_{i k 4}
\end{array}\right] .
\end{aligned}
$$


Similarly, for each subnetwork of the system (45) which satisfies Lemma 10, the Lyapunov-Krasovskii functional candidate can be chosen as

$$
\begin{aligned}
V_{i l}(t)= & z_{i}^{T}(t) E P_{i l 1} z_{i}(t)+\int_{t-h_{m}}^{t} z_{i}^{T}(s) Q_{i l} e^{\beta(t-s)} z_{i}(s) d s \\
& +\int_{t-h_{M}}^{t} z_{i}^{T}(s) R_{i l} e^{\beta(t-s)} z_{i}(s) d s \\
& +h_{m} \int_{-h_{m}}^{0} \int_{t+\theta}^{t} \dot{z}_{i}^{T}(s) U_{i l} e^{\beta(t-s)} \dot{z}_{i}(s) d s d \theta \\
& +h_{M} \int_{-h_{M}}^{0} \int_{t+\theta}^{t} y_{i}^{T}(s) S_{i l} e^{\beta(t-s)} y_{i}(s) d s d \theta \\
& +\delta \int_{-h_{M}}^{-h_{m}} \int_{t+\theta}^{t} \dot{z}_{i}^{T}(s) W_{i l} e^{\beta(t-s)} \dot{z}_{i}(s) d s d \theta
\end{aligned}
$$

where $i=2,3, \ldots, N, l \in S_{n}, P_{i l 1}, Q_{i l}, R_{i l}, U_{i l}, S_{i l}$, and $W_{i l}$ are positive definite matrices.

Consider the following piecewise Lyapunov-Krasovskii functional candidate:

$$
V_{i}(t)=V_{i \sigma(t)}, \quad i=2,3, \ldots, N, \sigma(t) \in M .
$$

From Lemmas 8 and 10, the following properties of the Lyapunov-Krasovskii functional candidate (49) are obtained.

(i) There exist $a, b>0$ such that

$$
\begin{aligned}
a\left\|z_{i}(t)\right\|^{2} & \leq V_{i k, i l}(t) \leq b\left\|z_{i}\left(t_{0}\right)\right\|_{\mathrm{cl}}^{2}, \\
& i=2,3, \ldots, N, k, l \in M .
\end{aligned}
$$

(ii) There exists a constant $\mu \geq 1$ such that

$$
V_{i k}(t) \leq \mu V_{i l}(t), \quad i=2,3, \ldots, N, k, l \in M .
$$

(iii) The Lyapunov functional candidate (49) satisfies

$$
V_{i}(t) \leq \begin{cases}e^{-\alpha\left(t-t_{0}\right)} V_{i k}\left(t_{0}\right), & \text { if } \sigma(t)=k \in S_{s}, \\ e^{\beta\left(t-t_{0}\right)} V_{i l}\left(t_{0}\right), & \text { if } \sigma(t)=l \in S_{n} .\end{cases}
$$

Now, for any piecewise constant switching signal $\sigma(t)$ and any $0 \leq t_{0}<t$, we let $T^{-}\left(t_{0}, t\right)\left(T^{+}\left(t_{0}, t\right)\right.$ resp. $)$ denote the total activation time of the synchronizable subnetworks (the ones of non-synchronizable subnetworks resp.) during $\left(t_{0}, t\right)$. Then, we choose a scalar $\alpha^{*} \in(0, \alpha)$ arbitrarily to propose the following switching law.

$(S 1)$ : Determine the switching signal $\sigma(t)$ so that

$$
\inf _{t \geq t_{0}} \frac{T^{-}\left(t_{0}, t\right)}{T^{+}\left(t_{0}, t\right)} \geq \frac{\beta+\alpha^{*}}{\alpha-\alpha^{*}}
$$

holds on time interval $\left(t_{0}, t\right)$. Meanwhile, we choose $\alpha^{*}<\alpha$ as the average dwell time scheme: for any $t>t_{0}$,

$$
N_{\sigma}\left(t_{0}, t\right) \leq N_{0}+\frac{t-t_{0}}{T_{a}}, \quad T_{a} \geq T_{a}^{*}=\frac{\ln \mu}{\alpha^{*}} .
$$

Theorem 11. For a given constant $\alpha>0, \beta>0$ and timevarying delay satisfying (3), suppose that the subnetwork $(1 \leq$ $k \leq r$ ) of switched network (1) satisfies the conditions of Lemma 8, and the others satisfy the conditions of Lemma 10. Assume that there exists $\mu \geq 1$ such that

$$
\begin{aligned}
& P_{i p 1} \leq \mu P_{i q 1}, \quad Q_{i p} \leq \mu Q_{i q}, \quad R_{i p} \leq \mu R_{i q}, \\
& U_{i p} \leq \mu U_{i q}, \quad S_{i p} \leq \mu S_{i q}, \quad W_{i p} \leq \mu W_{i q} \text {, } \\
& \forall p, q \in M \text {. }
\end{aligned}
$$

Then the synchronous manifold (5) of network (1) is locally exponentially stable for switching signal satisfying (53) and (54), and the state decay estimate is given by

$$
\left\|z_{i}(t)\right\| \leq \sqrt{\frac{c_{0} b_{i}}{a_{i}}} e^{-\lambda\left(t-t_{0}\right)}\left\|z_{i}\left(t_{0}\right)\right\|_{\mathrm{cl}}, \quad i=2,3, \ldots, N,
$$

where

$$
\begin{aligned}
c_{0}= & e^{N_{0} \ln \mu}, \quad \lambda=\frac{1}{2}\left(\alpha^{*}-\frac{\ln \mu}{T_{a}}\right), \\
a_{i}= & \min _{\forall k \in M}\left(\lambda_{\min }\left(P_{i k 1}\right)\right), \\
b_{i}= & \max _{\forall k \in M}\left(\lambda_{\max }\left(P_{i k 1}\right)\right)+h_{m_{\forall k \in M}} \max _{\forall}\left(\lambda_{\max }\left(Q_{i k}\right)\right) \\
& +h_{M} \max _{\forall k \in M}\left(\lambda_{\max }\left(R_{i k}\right)\right)+\frac{h_{m}^{2}}{2} \max _{\forall k \in M}\left(\lambda_{\max }\left(U_{i k}\right)\right) \\
& +\frac{h_{M}^{2}}{2} \max _{\forall k \in M}\left(\lambda_{\max }\left(S_{i k}\right)\right)+\frac{\delta^{2}}{2} \max _{\forall k \in M}\left(\lambda_{\max }\left(W_{i k}\right)\right) .
\end{aligned}
$$

Proof. Suppose that $t \in\left[t_{k}, t_{k+1}\right)$. For piecewise LyapunovKrasovskii functional candidate (49), along trajectory of network system (45), we have

$$
V_{i}(t) \leq \begin{cases}e^{-\alpha\left(t-t_{k}\right)} V_{i k}\left(t_{k}\right), & \text { if } \sigma(t)=k \in S_{s}, \\ e^{\beta\left(t-t_{k}\right)} V_{i l}\left(t_{k}\right), & \text { if } \sigma(t)=l \in S_{n} .\end{cases}
$$

Since $V_{i \sigma\left(t_{k}\right)}\left(t_{k}\right) \leq \mu V_{i \sigma\left(t_{k}^{-}\right)}\left(t_{k}^{-}\right)$is true from (51) at the switching point $t_{k}$, where $t_{k}^{-}=\lim _{t \rightarrow t_{k}} t$ and from $k=$ $N_{\sigma}\left(t_{0}, t\right) \leq N_{0}+\left(\left(t-t_{0}\right) / T_{a}\right)$, we obtain

$$
\begin{aligned}
V_{i}(t) \leq & e^{\beta T^{+}\left(t_{k}, t\right)-\alpha T^{-}\left(t_{k}, t\right)} V_{i \sigma\left(t_{k}\right)}\left(t_{k}\right) \\
\leq & e^{\beta T^{+}\left(t_{k}, t\right)-\alpha T^{-}\left(t_{k}, t\right)} \mu V_{i \sigma\left(t_{k}^{-}\right)}\left(t_{k}^{-}\right) \\
\leq & e^{\beta T^{+}\left(t_{k}, t\right)-\alpha T^{-}\left(t_{k}, t\right)} \mu e^{\beta T^{+}\left(t_{k-1}, t_{k}\right)-\alpha T^{-}\left(t_{k-1}, t_{k}\right)} \\
& \times V_{i \sigma\left(t_{k-1}\right)}\left(t_{k-1}\right) \\
\leq & \mu e^{\beta T^{+}\left(t_{k-1}, t\right)-\alpha T^{-}\left(t_{k-1}, t\right)} V_{i \sigma\left(t_{k-1}\right)}\left(t_{k-1}\right) \\
& \vdots \\
\leq & \mu^{k} e^{\beta T^{+}\left(t_{0}, t\right)-\alpha T^{-}\left(t_{0}, t\right)} V_{i \sigma\left(t_{0}\right)}\left(t_{0}\right) \\
\leq & e^{\beta T^{+}\left(t_{0}, t\right)-\alpha T^{-}\left(t_{0}, t\right)+\left(N_{0}+\left(\left(t-t_{0}\right) / T_{a}\right)\right) \ln \mu} V_{i \sigma\left(t_{0}\right)}\left(t_{0}\right) .
\end{aligned}
$$


TABLE 1: Comparison of the maximum value $h_{M}$ for the asymptotic stability of system (65) by different methods.

\begin{tabular}{lccccc}
\hline & $c$ & 0.3 & 0.4 & 0.5 & 0.6 \\
\hline \multirow{3}{*}{$h_{m}=0$} & Li et al. 2008 [8] & 0.960 & 0.710 & 0.562 & 0.464 \\
& Yue and Li 2010 [12] & 1.345 & 0.950 & 0.731 & 1.0983 \\
$h_{m}=0.1$ & Ours & 1.8894 & 1.3923 & 0.587 \\
& Yue and Li 2010 [12] & 1.354 & 0.951 & 1.4171 & 0.9049 \\
$h_{m}=0.5$ & Ours & 1.9158 & 0.967 & 0.587 \\
& Yue and Li 2010 [12] & 1.389 & 1.6165 & 0.740 & 0.9276 \\
\hline
\end{tabular}

Under the switching law $(S 1)$ for any $t_{0}$, $t$, we have

$$
\begin{aligned}
\beta T^{+}\left(t_{0}, t\right)-\alpha T^{-}\left(t_{0}, t\right) & \leq-\alpha^{*}\left(T^{+}\left(t_{0}, t\right)+T^{-}\left(t_{0}, t\right)\right) \\
& =-\alpha^{*}\left(t-t_{0}\right)
\end{aligned}
$$

Thus,

$$
\begin{aligned}
V_{i}(t) & \leq e^{N_{0} \ln \mu} e^{-\left(\alpha^{*}-\left((\ln \mu) / T_{a}\right)\right)\left(t-t_{0}\right)} V_{i \sigma\left(t_{0}\right)}\left(t_{0}\right) \\
& \leq c_{0} e^{-2 \lambda\left(t-t_{0}\right)} V_{i \sigma\left(t_{0}\right)}\left(t_{0}\right),
\end{aligned}
$$

where $c_{0}=e^{N_{0} \ln \mu}, \lambda=(1 / 2)\left(\alpha^{*}-\left((\ln \mu) / T_{a}\right)\right)$. According to (49), we have

$$
a_{i}\left\|z_{i}(t)\right\|^{2} \leq V_{i}(t), \quad V_{i \sigma\left(t_{0}\right)}\left(t_{0}\right) \leq b_{i}\left\|z_{i}\left(t_{0}\right)\right\|_{\mathrm{cl}}^{2}
$$

Combining (61) and (62) leads to

$$
\left\|z_{i}(t)\right\|^{2} \leq \frac{1}{a_{i}} V_{i}(t) \leq \frac{b_{i}}{a_{i}} c_{0} e^{-2 \lambda\left(t-t_{0}\right)}\left\|z_{i}\left(t_{0}\right)\right\|_{\mathrm{cl}}^{2} .
$$

Therefore,

$$
\left\|z_{i}(t)\right\| \leq \sqrt{\frac{c_{0} b_{i}}{a_{i}}} e^{-\lambda\left(t-t_{0}\right)}\left\|z_{i}\left(t_{0}\right)\right\|_{\mathrm{cl}} .
$$

which means that synchronous solution to (1) is locally exponentially stable. The proof is completed.

\section{Numerical Examples}

In this section, we give examples to show the effectiveness of theoretical results obtained in Lemma 8 and Theorem 11.

Example 12. Consider a nonswitched network model with 5 nodes, where each node is a three-dimensional stable linear system described by

$$
\begin{aligned}
& \dot{x}_{i 1}(t)=-x_{i 1}(t) \\
& \dot{x}_{i 2}(t)=-2 x_{i 2}(t) \\
& \dot{x}_{i 3}(t)=-3 x_{i 3}(t),
\end{aligned}
$$

which is asymptotically stable at the equilibrium point $s(t)=$ 0 , and its Jacobin matrices are $J(t)=\operatorname{diag}\{-1,-2,-3\}$,
$J_{h}(t-h(t))=0$. Assume that the inner-coupling matrix is $A=$ $\operatorname{diag}\{1,1,1\}$, and the outer-coupling matrix is given by the following irreducible symmetric matrix satisfying condition (2):

$$
G=\left[\begin{array}{ccccc}
-2 & 1 & 0 & 0 & 1 \\
1 & -3 & 1 & 1 & 0 \\
0 & 1 & -2 & 1 & 0 \\
0 & 1 & 1 & -3 & 1 \\
1 & 0 & 0 & 1 & -2
\end{array}\right]
$$

The eigenvalues of $G$ are $\lambda_{i}=\{0,-1.382,-2.382,-3.618,-$ $4.618\}, i=1,2, \ldots, 5$. Therefore, if the delayed subsystem in (17) is asymptotically stable, then the synchronized state $s(t)$ is asymptotically stable.

In this example, we have $\widetilde{A}_{i}=\operatorname{diag}\{-1,-2,-3\}, \widetilde{B}_{i}=$ $\operatorname{diag}\left\{c \lambda_{i}, c \lambda_{i}, c \lambda_{i}\right\}$, and $i=2, \ldots, 5$. Let us decompose the matrix $\widetilde{B}_{i}=\widetilde{B}_{i 1}+\widetilde{B}_{i 2}$, where

$$
\begin{gathered}
\widetilde{B}_{i 1}=\left[\begin{array}{ccc}
0.39 c \lambda_{i} & 0 & 0 \\
0 & 0.39 c \lambda_{i} & 0 \\
0 & 0 & 0.39 c \lambda_{i}
\end{array}\right], \\
\widetilde{B}_{i 2}=\left[\begin{array}{ccc}
0.61 c \lambda_{i} & 0 & 0 \\
0 & 0.61 c \lambda_{i} & 0 \\
0 & 0 & 0.61 c \lambda_{i}
\end{array}\right], \\
\\
\quad i=2, \ldots, 5 .
\end{gathered}
$$

By using Lemma 8, we will give the maximum upper bounds $h_{M}$ of the time-varying delay for different lower bounds $h_{m}$ and coupling strength $c$. Applying Lemma 8 with $\alpha=0$, we obtain the maximum upper bound of delays $h_{M}$ as shown in Table 1 . We see that, Lemma 8 provides a less conservative result than those obtained via the methods of $[8,12]$. When $h_{m} \neq 0$ especially, the result in [8] is not discussed while Lemma 8 in this paper also considers the case $h_{m} \neq 0$. Note that we use MATLAB LMI Control Toolbox in order to solve the LMI in (24) and (25).

The numerical simulations are carried out using the explicit Runge-Kutta-like method (dde45), interpolation and extrapolation by spline of the third order. Figure 1 shows the synchronization state curves $x_{i 1}(t), x_{i 2}(t), x_{i 3}(t)$, 


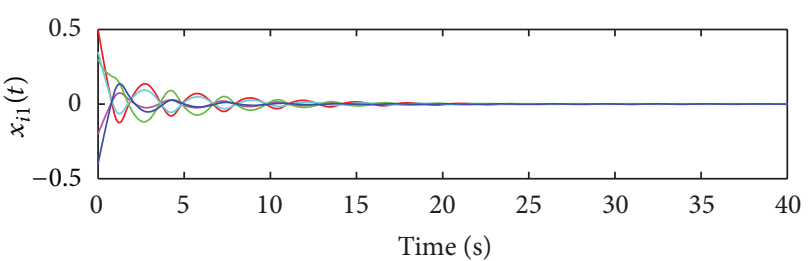

(a)

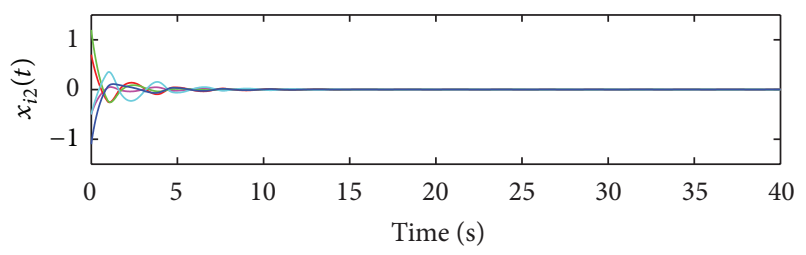

(b)

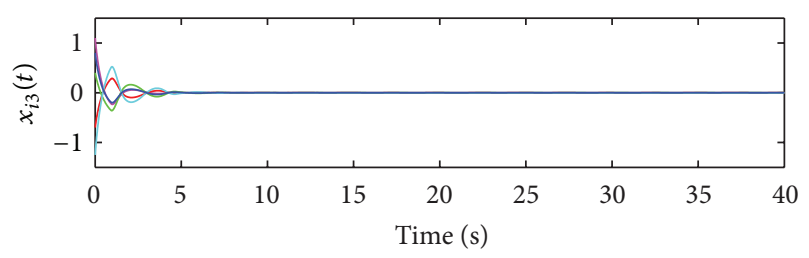

(c)

Figure 1: Synchronization state curves for the dynamic nodes delayed network (65) with $c=0.5$ and $h(t)=0.5+0.715|\cos t|$.

$i=1,2, \ldots, 5$ with $c_{1}=0.5, h(t)=0.5+0.715|\cos t|$ and the initial function

$$
\begin{aligned}
& \phi_{1}(t)=[-0.2 \cos t,-0.5 \cos t, 1.1 \cos t], \\
& \phi_{2}(t)=[0.5 \cos t, 0.7 \cos t,-0.7 \cos t], \\
& \phi_{3}(t)=[0.3 \cos t, 1.2 \cos t, 0.4 \cos t], \\
& \phi_{4}(t)=[0.35 \cos t,-0.5 \cos t,-1.25 \cos t], \\
& \phi_{5}(t)=[-0.4 \cos t,-1.1 \cos t, 0.8 \cos t] .
\end{aligned}
$$

Example 13. Consider a linear switched network model with 5 nodes where each node is described by the following delayed dynamical system:

$$
\begin{aligned}
& \dot{x}_{i 1}(t)=-1 x_{i 1}(t)-x_{i 1}(t-h(t)) \\
& \dot{x}_{i 2}(t)=-2 x_{i 2}+x_{i 1}(t-h(t))-x_{i 2}(t-h(t)) \\
& \dot{x}_{i 3}(t)=-1.5 x_{i 3}-x_{i 3}(t-h(t)),
\end{aligned}
$$

which is asymptotically stable at the equilibrium point $s(t)=$ $0, s(t-h(t))=0$. Jacobian matrices $J(t)$ and $J_{h}(t-h(t))$ are

$$
J(t)=\left[\begin{array}{ccc}
-1 & 0 & 0 \\
0 & -2 & 0 \\
0 & 0 & -1.5
\end{array}\right], \quad J_{h}(t-h(t))=\left[\begin{array}{ccc}
-1 & 0 & 0 \\
1 & -1 & 0 \\
0 & 0 & -1
\end{array}\right] .
$$

Assume that the inner-coupling matrices are $A_{1}=$ $\operatorname{diag}\{0.5,0.5,0.5\}, A_{2}=\operatorname{diag}\{-1,-1,-1\}$ and the coupling strengths are $c_{1}=0.3$ and $c_{2}=0.6$, and the outer-coupling matrices are

$$
\begin{aligned}
& G_{1}=\left[\begin{array}{ccccc}
-2 & 1 & 0 & 0 & 1 \\
1 & -3 & 1 & 1 & 0 \\
0 & 1 & -2 & 1 & 0 \\
0 & 1 & 1 & -3 & 1 \\
1 & 0 & 0 & 1 & -2
\end{array}\right], \\
& G_{2}=\left[\begin{array}{ccccc}
-4 & 1 & 1 & 1 & 1 \\
1 & -4 & 1 & 1 & 1 \\
1 & 1 & -4 & 1 & 1 \\
1 & 1 & 1 & -4 & 1 \\
1 & 1 & 1 & 1 & -4
\end{array}\right] .
\end{aligned}
$$

The eigenvalues of $G_{1}$ and $G_{2}$ are $\lambda_{i 1}=\{0,-1.382,-2.382,-$ $3.618,-4.618\}, \lambda_{i 2}=\{0,-5,-5,-5,-5\}$, and $i=1,2, \ldots, 5$, respectively. Obviously, there exists a unitary matrix $U$ which diagonalizes $G_{1}$ and $G_{2}$ simultaneously. The subnetwork associated with $\left\{c_{1}, A_{1}, G_{1}\right\}$ is synchronizable and the subnetwork associated with $\left\{c_{2}, A_{2}, G_{2}\right\}$ is non-synchronizable.

From the above conditions, we obtain matrices

$$
\begin{aligned}
\widetilde{A}_{i} & =\left[\begin{array}{ccc}
-1 & 0 & 0 \\
0 & -2 & 0 \\
0 & 0 & -1.5
\end{array}\right], \\
\widetilde{B}_{i k} & =\left[\begin{array}{ccc}
-1+c_{k} a_{11}^{k} \lambda_{i k} & 0 & 0 \\
1 & -1+c_{k} a_{22}^{k} \lambda_{i k} & 0 \\
0 & 0 & -1+c_{k} a_{33}^{k} \lambda_{i k}
\end{array}\right],
\end{aligned}
$$

$i=2, \ldots, 5$, and $k=1,2$. Let us decompose the matrix $\widetilde{B}_{i k}=$ $\widetilde{B}_{i k 1}+\widetilde{B}_{i k 2}$, where

$$
\begin{array}{ll}
\widetilde{B}_{i 11}=0.93 \widetilde{B}_{i 1}, & \widetilde{B}_{i 12}=0.07 \widetilde{B}_{i 1}, \\
\widetilde{B}_{i 21}=0.86 \widetilde{B}_{i 2}, & \widetilde{B}_{i 22}=0.14 \widetilde{B}_{i 2}, \quad i=2, \ldots, 5 .
\end{array}
$$

Given $h_{1}=0.3, h_{2}=0.68, \alpha=1.25$, and $\beta=0.6$, it is found that LMIs (24), (25), (42), and (42) have feasible solutions. We may choose $\mu=1.35$. Let $\alpha^{*}=0.55<$ $\alpha$. Then, from the switching law $(S 1)$, it is required that $\inf _{t \geq t_{0}}\left(T^{-}\left(t_{0}, t\right) / T^{+}\left(t_{0}, t\right)\right) \geq\left(\beta+\alpha^{*}\right) /\left(\alpha-\alpha^{*}\right)=1.6429$ and the average dwell time is computed as $T_{a} \geq T_{a}^{*}=(\ln \mu) / \alpha^{*}=$ 0.5456 .

Let $h(t)=0.3+0.38|\sin t|$ and the initial function

$$
\begin{gathered}
\phi_{1}(t)=[-0.4 \cos t,-0.5 \cos t, 1.1 \cos t], \\
\phi_{2}(t)=[0.5 \cos t, 0.7 \cos t,-0.7 \cos t], \\
\phi_{3}(t)=[0.89 \cos t, 1.2 \cos t, 0.4 \cos t], \\
\phi_{4}(t)=[1.35 \cos t,-1.5 \cos t,-1.25 \cos t], \\
\phi_{5}(t)=[-0.8 \cos t,-1.1 \cos t, 0.8 \cos t] .
\end{gathered}
$$




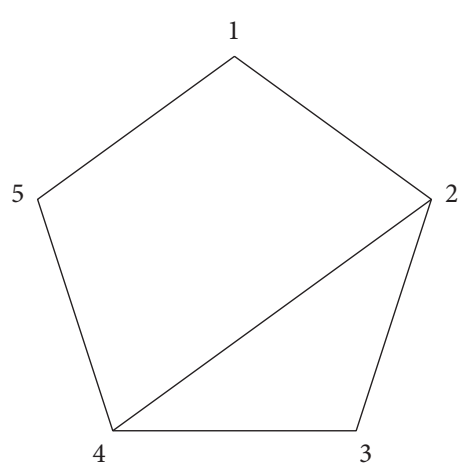

$G_{1}$

(a)

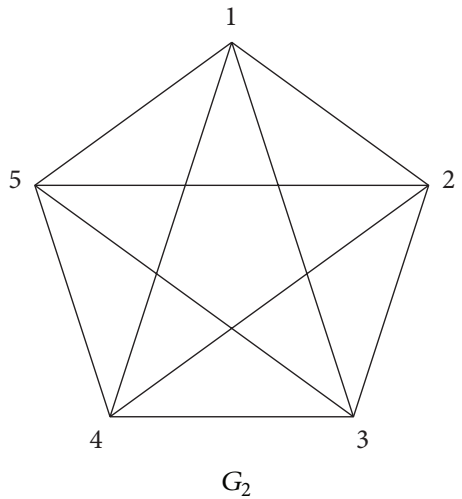

(b)

FIGURE 2: The structure of complex networks with $N=5$.

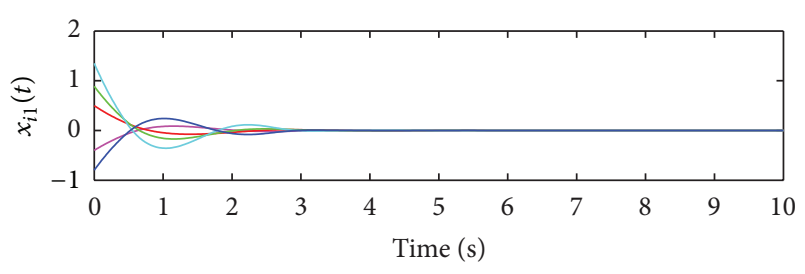

(a)

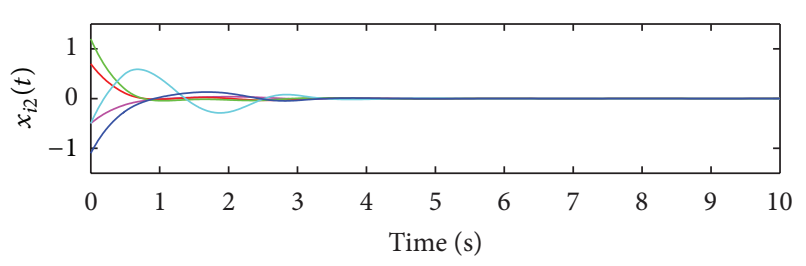

(b)

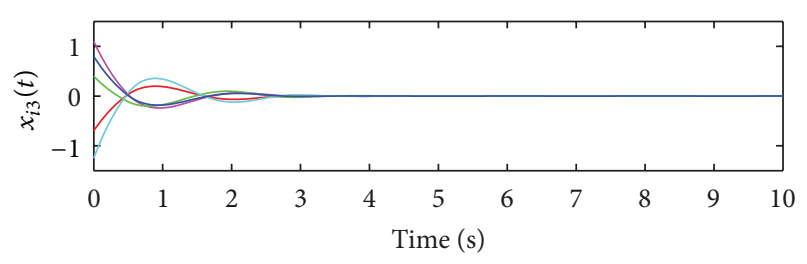

(c)

Figure 3: Synchronization state curves for the synchronizable subnetwork (69).

Figure 2 shows the structure of complex networks. Figures 3 and 4 show the synchronization state curves of the synchronizable subnetwork and non-synchronizable subnetwork, respectively. Figure 5 shows the synchronization state curves $x_{i 1}(t), x_{i 2}(t), x_{i 3}(t), i=1,2, \ldots, 5$ of switched network with $\sigma(t)$ satisfying (S1). Figure 6 shows the switching $\sigma(t)$ of the switched delay network with average dwell time. We see that the synchronization state converges to zero under the above conditions.

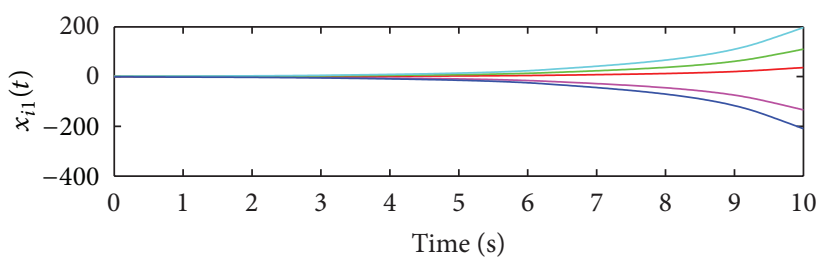

(a)

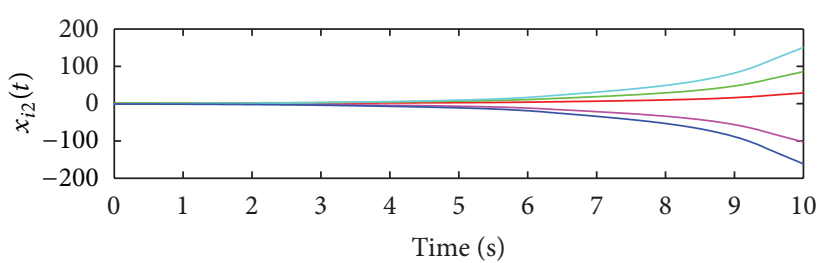

(b)

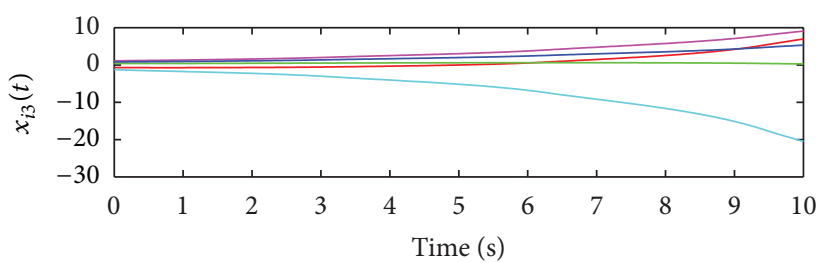

(c)

FIGURE 4: Synchronization state curves for the nonsynchronizable subnetwork (69).

Example 14. We consider nonlinear switched network model with 5 nodes in which each node is a Lorenz chaotic system with time-varying delay described by [35] as

$$
\begin{aligned}
& \dot{x}_{i 1}(t)=a\left(x_{i 2}(t)-x_{i 1}(t)\right), \\
& \dot{x}_{i 2}(t)=c x_{i 1}-x_{i 2}(t)-x_{i 1}(t) x_{i 3}(t-h(t)), \\
& \dot{x}_{i 3}(t)=x_{i 1}(t) x_{i 2}(t-h(t))-b x_{i 3}(t-h(t)),
\end{aligned}
$$




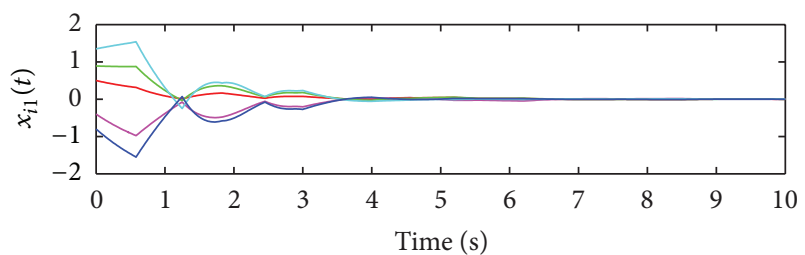

(a)

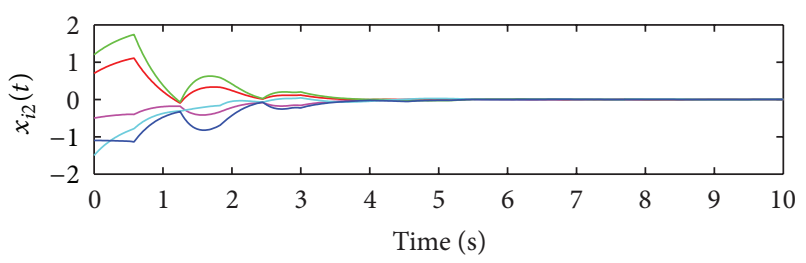

(b)

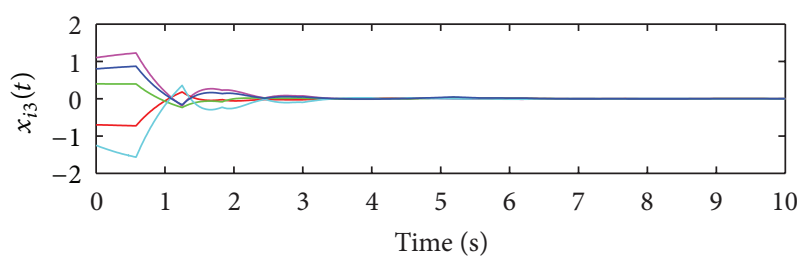

(c)

FIGURE 5: Synchronization state curves for the switched delay network (69).

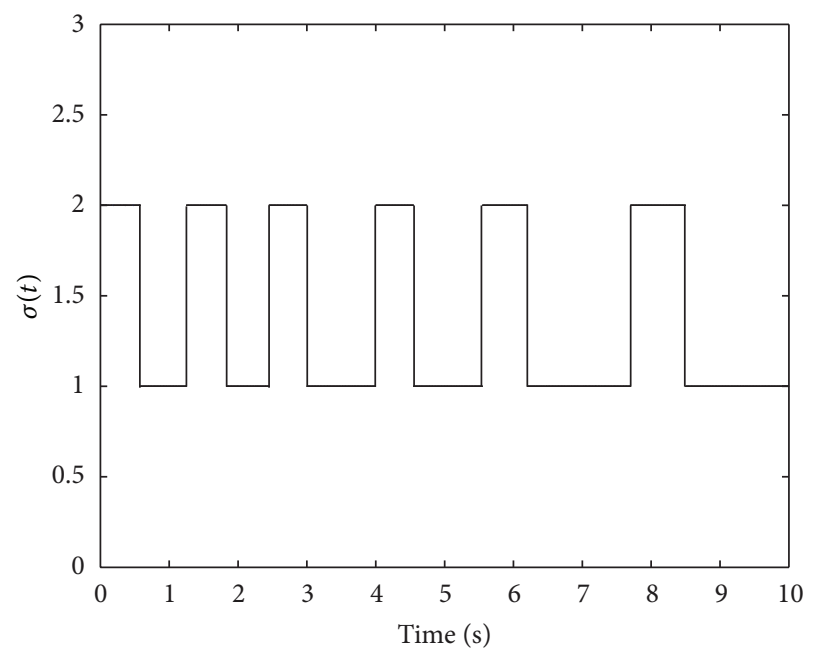

FIGURE 6: The switching signal $\sigma(t)$ of the switched delay network (69) with average dwell time.

where $a=10, b=1.3$, and $c=-28$. It is asymptotically stable at the equilibrium point $s(t)=0, s(t-h(t))=0$ and its Jacobian matrices are

$$
J(t)=\left[\begin{array}{ccc}
-10 & 10 & 0 \\
-28 & -1 & 0 \\
0 & 0 & 0
\end{array}\right], \quad J_{h}(t-h(t))=\left[\begin{array}{ccc}
0 & 0 & 0 \\
0 & 0 & -1 \\
0 & 0 & -1.3
\end{array}\right]
$$

Assume that the inner-coupling matrices are $A_{1}=$ $\operatorname{diag}\{0.4,0.4,0.4\}$ and $A_{2}=\operatorname{diag}\{-0.2,-0.2,-0.2\}$, the coupling strengths $c_{1}=0.5$, and $c_{2}=0.3$, and the outercoupling matrices $G_{1}$, and $G_{2}$ are the same as in Example 13. The subnetwork associated with $\left\{c_{1}, A_{1}, G_{1}\right\}$ is synchronizable and the subnetwork associated with $\left\{c_{2}, A_{2}, G_{2}\right\}$ is nonsynchronizable.

From the above conditions, we obtain

$$
\begin{gathered}
\widetilde{A}_{i}=\left[\begin{array}{ccc}
-10 & 10 & 0 \\
-28 & -1 & 0 \\
0 & 0 & 0
\end{array}\right], \\
\widetilde{B}_{i k}=\left[\begin{array}{ccc}
c_{k} a_{11}^{k} \lambda_{i k} & 0 & 0 \\
0 & c_{k} a_{22}^{k} \lambda_{i k} & -1 \\
0 & 0 & -1.3+c_{k} a_{33}^{k} \lambda_{i k}
\end{array}\right],
\end{gathered}
$$

$i=2, \ldots, 5$, and $k=1,2$. Let us decompose the matrix $\widetilde{B}_{i k}=$ $\widetilde{B}_{i k 1}+\widetilde{B}_{i k 2}$, where

$$
\begin{gathered}
\widetilde{B}_{i 11}=0.8 \widetilde{B}_{i 1}, \quad \widetilde{B}_{i 12}=0.2 \widetilde{B}_{i 1}, \\
\widetilde{B}_{i 21}=0.86 \widetilde{B}_{i 2}, \quad \widetilde{B}_{i 22}=0.14 \widetilde{B}_{i 2}, \quad i=2, \ldots, 5 .
\end{gathered}
$$

Given $h_{1}=0.2, h_{2}=0.55, \alpha=0.9$, and $\beta=0.3$, it is found that LMIs (24), (25), (42), and (42) have feasible solutions. We may choose $\mu=1.253$. Let $\alpha^{*}=0.45<$ $\alpha$. Then, from the switching law $(S 1)$, it is required that $\inf _{t \geq t_{0}}\left(T^{-}\left(t_{0}, t\right) / T^{+}\left(t_{0}, t\right)\right) \geq\left(\beta+\alpha^{*}\right) /\left(\alpha-\alpha^{*}\right)=1.6667$ and the average dwell time is computed as $T_{a} \geq T_{a}^{*}=(\ln \mu) / \alpha^{*}=$ 0.5012 .

Let $h(t)=0.2+0.35|\cos t|$ and the initial function

$$
\begin{gathered}
\phi_{1}(t)=[15 \cos t, 20 \cos t, 5 \cos t], \\
\phi_{2}(t)=[5 \cos t, 15 \cos t,-2 \cos t], \\
\phi_{3}(t)=[10 \cos t, 5 \cos t, 3 \cos t], \\
\phi_{4}(t)=[20 \cos t, 10 \cos t,-1 \cos t], \\
\phi_{5}(t)=[15 \cos t, 20 \cos t, 8 \cos t] .
\end{gathered}
$$

Figures 7 and 8 show the synchronization errors between the states of node $i$ and node $i+1, e_{i j}(t)=x_{i j}-x_{(i+1) j}, i=1, \ldots, 4$, and $j=1, \ldots, 3$ of the synchronizable subnetwork and nonsynchronizable subnetwork, respectively. Figure 9 shows the synchronization errors between the states of node $i$ and node $i+1$ of switched network with $\sigma(t)$ satisfying $(S 1)$. Figure 10 shows the switching $\sigma(t)$ of the switched delay network with average dwell time. We see that the synchronization state converges to zero under the above conditions.

Remark 15. The advantage of Examples 13 and 14 is the lower bound of the delay $h_{m} \neq 0$. Moreover, in these examples we still investigate interval time-varying delays in the dynamical nodes and the switched coupling term simultaneously, hence the synchronization conditions derived in [33] cannot be applied to these examples. 


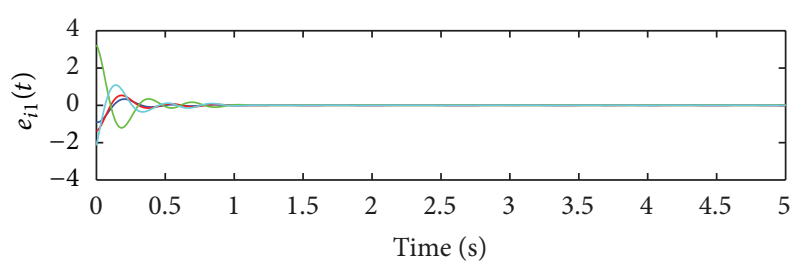

(a)

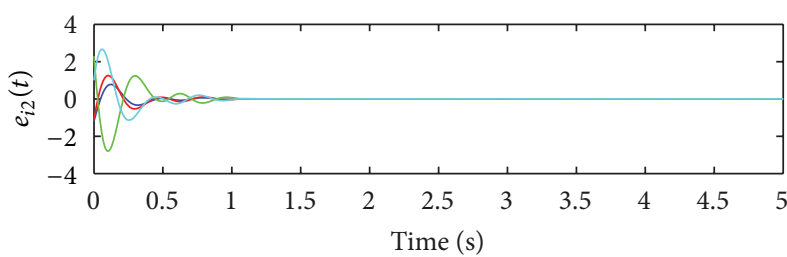

(b)

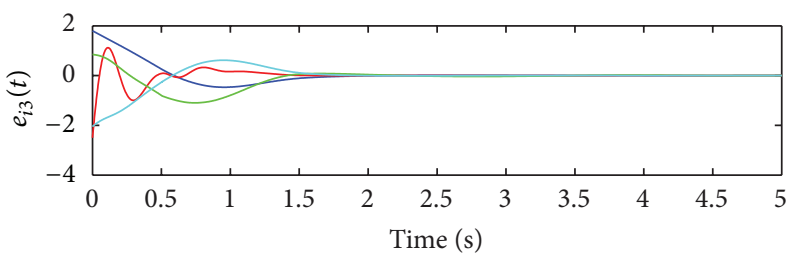

(c)

FIgURE 7: Synchronization errors between the states of node $i$ and node $i+1$ for the synchronizable subnetwork (75).

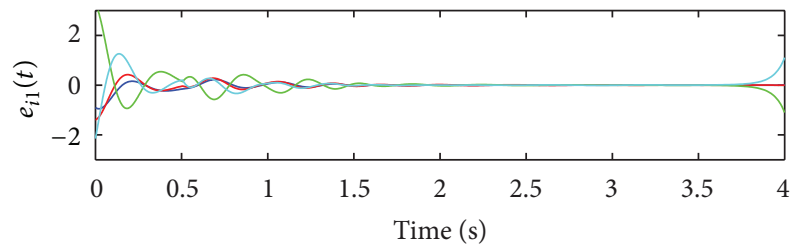

(a)

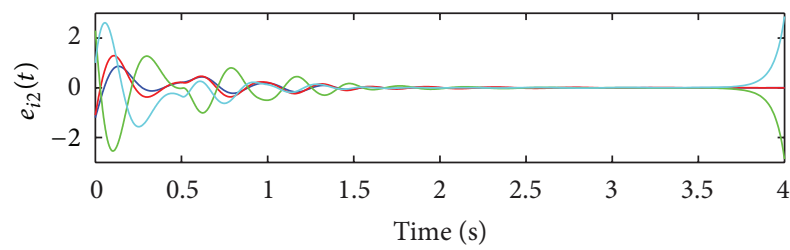

(b)



(c)

FIGURE 8: Synchronization errors between the states of node $i$ and node $i+1$ for the non-synchronizable subnetwork (75).

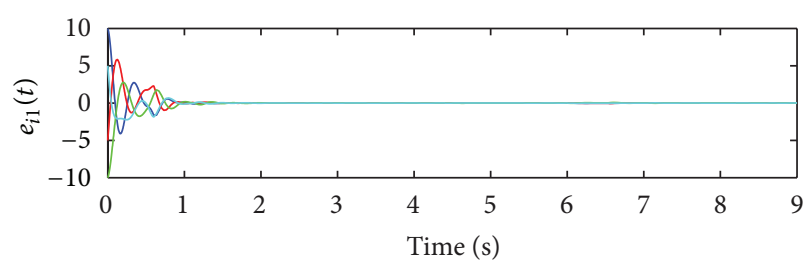

(a)



(b)

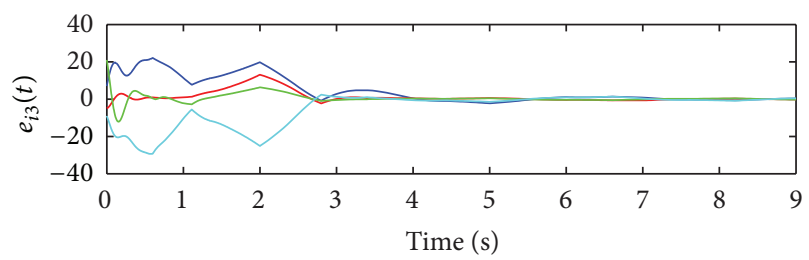

(c)

FIgURE 9: Synchronization errors between the states of node $i$ and node $i+1$ for the switched delay network (75).

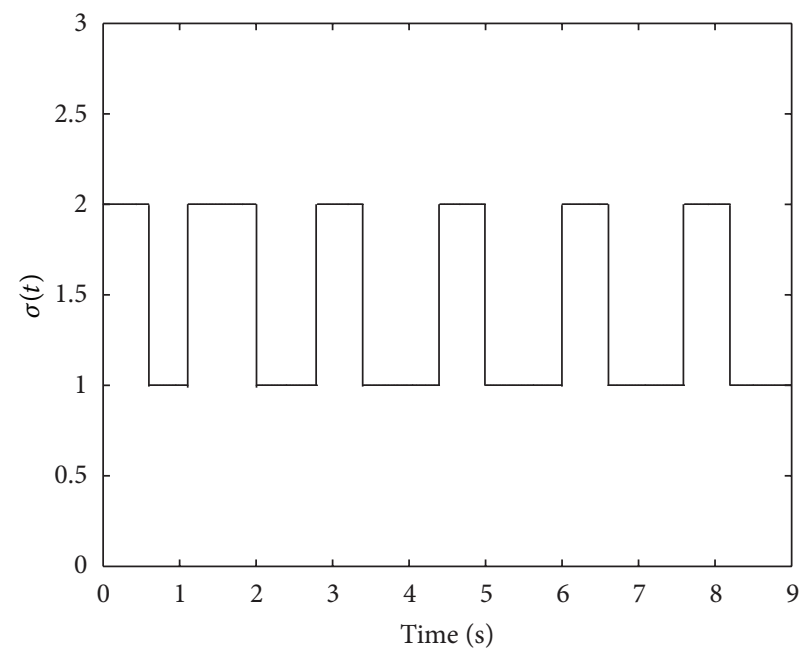

FIGURE 10: The switching signal $\sigma(t)$ of the switched delay network (75) with average dwell time.

\section{Conclusion}

In this paper, the synchronization problem has been investigated for complex dynamical networks with interval timevarying and switched coupling delays. Both interval timevarying delays in dynamical nodes and interval time-varying delays in switched couplings have been considered. We transformed the synchronization problem of the switched 
network into the stability analysis of linear switched systems. By using the average dwell time approach and piecewise Lyapunov-Krasovskii functionals which are constructed based on the descriptor model transformation and decomposition technique of coefficient matrix, new delay-dependent synchronization criteria was derived in terms of linear matrix inequalities. Numerical examples were given to illustrate that the derived criteria are less conservative than some existing results.

\section{Acknowledgments}

The first author is supported by the Center of Excellence in Mathematics, Thailand, and Commission for Higher Education. The second author is supported by the Center of Excellence in Mathematics, Thailand and Commission for Higher Education, Thailand. The authors also wish to thank the National Research University Project under Thailand's Office of the Higher Education Commission for financial support. They would like to thank Professor Xinzhi Liu for the valuable comments and suggestions.

\section{References}

[1] M. Faloutsos, P. Faloutsos, and C. Faloutsos, "On power-law relationships of the Internet topology," Computer Communication Review, vol. 29, pp. 251-263, 1999.

[2] R. Albert, H. Jeong, and A. L. BarabVasi, "Diameter of the world wide web," Nature, vol. 401, pp. 130-131, 1999.

[3] R. J. Williams and N. D. Martinez, "Simple rules yield complex food webs," Nature, vol. 404, pp. 180-183, 2000.

[4] H. Jeong, B. Tombor, R. Albert, Z. Oltvai, and A. L. BarabVasi, "The large-scale organization of metabolic network," Nature, vol. 407, pp. 651-653, 2000.

[5] S. Wassrman and K. Faust, Social Network Analysis, Cambridge University Press, Cambridge, UK, 1994.

[6] S. H. Strogatz, "Exploring complex networks," Nature, vol. 410, pp. 268-276, 2001.

[7] C. Li and G. Chen, "Synchronization in general complex dynamical networks with coupling delays," Physica A, vol. 343, no. 1-4, pp. 263-278, 2004.

[8] K. Li, S. Guan, X. Gong, and C.-H. Lai, "Synchronization stability of general complex dynamical networks with timevarying delays," Physics Letters A, vol. 372, no. 48, pp. 7133-7139, 2008.

[9] T. Liu and J. Zhao, "Synchronization of complex switched delay dynamical networks with simultaneously diagonalizable coupling matrices," Journal of Control Theory and Applications, vol. 6, no. 4, pp. 351-356, 2008.

[10] X. F. Wang and G. Chen, "Pinning control of scale-free dynamical networks," Physica A, vol. 310, no. 3-4, pp. 521-531, 2002.

[11] X. F. Wang and G. Chen, "Synchronization in scale-free dynamical networks: robustness and fragility," IEEE Transactions on Circuits and Systems, vol. 49, no. 1, pp. 54-62, 2002.

[12] D. Yue and H. Li, "Synchronization stability of continuous/discrete complex dynamical networks with interval timevarying delays," Neurocomputing, vol. 73, pp. 809-819, 2010.

[13] X. F. Wang and G. Chen, "Synchronizationin small-world dynamical networks," International Journal of Bifurcation and Chaos, vol. 12, pp. 187-192, 2002.
[14] K. Gu, V. L. Kharitonov, and J. Chen, Stability of Time-Delay System, Birkhauser, Boston, Mass, USA, 2003.

[15] Q.-L. Han, "Robust stability for a class of linear systems with time-varying delay and nonlinear perturbations," Computers \& Mathematics with Applications, vol. 47, no. 8-9, pp. 1201-1209, 2004.

[16] Q.-L. Han and K. Gu, "Stability of linear systems with timevarying delay: a generalized discretized Lyapunov functional approach," Asian Journal of Control, vol. 3, pp. 170-180, 2001.

[17] X. Jiang and Q.-L. Han, "On $H_{\infty}$ control for linear systems with interval time-varying delay," Automatica, vol. 41, no. 12, pp. 2099-2106, 2005.

[18] P. Park, "A delay-dependent stability criterion for systems with uncertain time-invariant delays," IEEE Transactions on Automatic Control, vol. 44, no. 4, pp. 876-877, 1999.

[19] H.-Y. Shao, "New delay-dependent stability criteria for systems with interval delay," Automatica, vol. 45 , no. 3, pp. 744-749, 2009.

[20] S. Xu, J. Lam, and Y. Zou, "Further results on delay-dependent robust stability conditions of uncertain neutral systems," International Journal of Robust and Nonlinear Control, vol. 15, no. 5, pp. 233-246, 2005.

[21] K. Gu and S.-I. Niculescu, "Additional dynamics in transformed time-delay systems," IEEE Transactions on Automatic Control, vol. 45, no. 3, pp. 572-575, 2000.

[22] Q.-L. Han, "A descriptor system approach to robust stability of uncertain neutral systems with discrete and distributed delays," Automatica, vol. 40, no. 10, pp. 1791-1796, 2004.

[23] C. Peng and Y.-C. Tian, "Delay-dependent robust stability criteria for uncertain systems with interval time-varying delay," Journal of Computational and Applied Mathematics, vol. 214, no. 2, pp. 480-494, 2008.

[24] J. Tian, L. Xiong, J. Liu, and X. Xie, "Novel delay-dependent robust stability criteria for uncertain neutral systems with timevarying delay," Chaos, Solitons \& Fractals, vol. 40, no. 4, pp. 1858-1866, 2009.

[25] Q.-K. Li, G. M. Dimirovski, and J. Zhao, "A Solution to the tracking control problem for switched linear systems with timevarying delays," in Proceedings of the 47th IEEE Conference on Decision and Control, pp. 5348-5353, December 2008.

[26] D. Liberzon, Switching in Systems and Control, Birkhäuser, Boston, Mass, USA, 2003.

[27] J. Liu, X. Liu, and W.-C. Xie, "Delay-dependent robust control for uncertain switched systems with time-delay," Nonlinear Analysis. Hybrid Systems, vol. 2, no. 1, pp. 81-95, 2008.

[28] D. Liu, S. Zhong, X. Liu, and Y. Huang, "Stability analysis for uncertain switched neutral systems with discrete time-varying delay: a delay-dependent method," Mathematics and Computers in Simulation, vol. 80, no. 2, pp. 436-448, 2009.

[29] Z. D. Sun and S. S. Ge, Switched Linear Systems Control and Design, Springer, New York, NY, USA, 2004.

[30] X.-M. Sun, D. Wang, W. Wang, and G.-H. Yang, "Stability analysis and $\mathrm{L}_{2}$-gain of switched delay systems with stable and unstable subsystems," IEEE International Symposium on Intelligent Control, pp. 208-213, 2007.

[31] G. Zhai, B. Hu, K. Yasuda, and A. N. Michel, "Piecewise Lyapunov functions for switched systems with average dwell time," Asian Journal of Control, vol. 2, pp. 192-197, 2000.

[32] G. Zhai, B. Hu, K. Yasuda, and A. N. Michel, "Stability analysis of switched systems with stable and unstable subsystems: an average dwell time approach," International Journal of Systems Science, vol. 32, no. 8, pp. 1055-1061, 2001. 
[33] T. Liu, J. Zhao, and D. J. Hill, "Exponential synchronization of complex delayed dynamical networks with switching topology," IEEE Transactions on Circuits and Systems. I. Regular Papers, vol. 57, no. 11, pp. 2967-2980, 2010.

[34] R. A. Horn and C. R. Johnson, Matrix Analysis, Cambridge University Press, Cambridge, UK, 1985.

[35] Q. J. Zhang, J. A. Lu, J. H. Lu, and C. K. Tse, "Adaptive feedback synchronization of a general complex dynamical network with delayed nodes," IEEE Transactions on Circuits and Systems II: Express Briefs, vol. 55, pp. 183-187, 2008. 


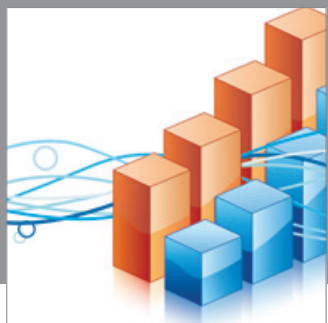

Advances in

Operations Research

mansans

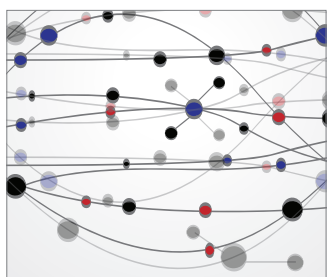

The Scientific World Journal
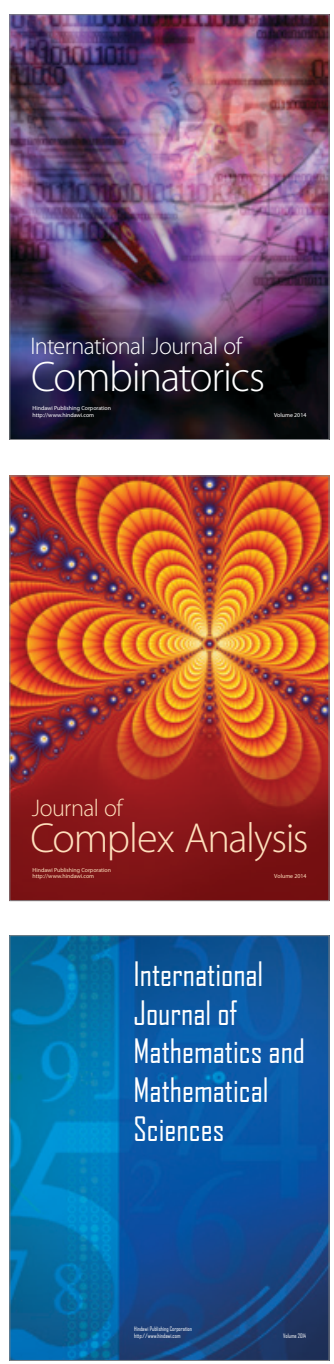
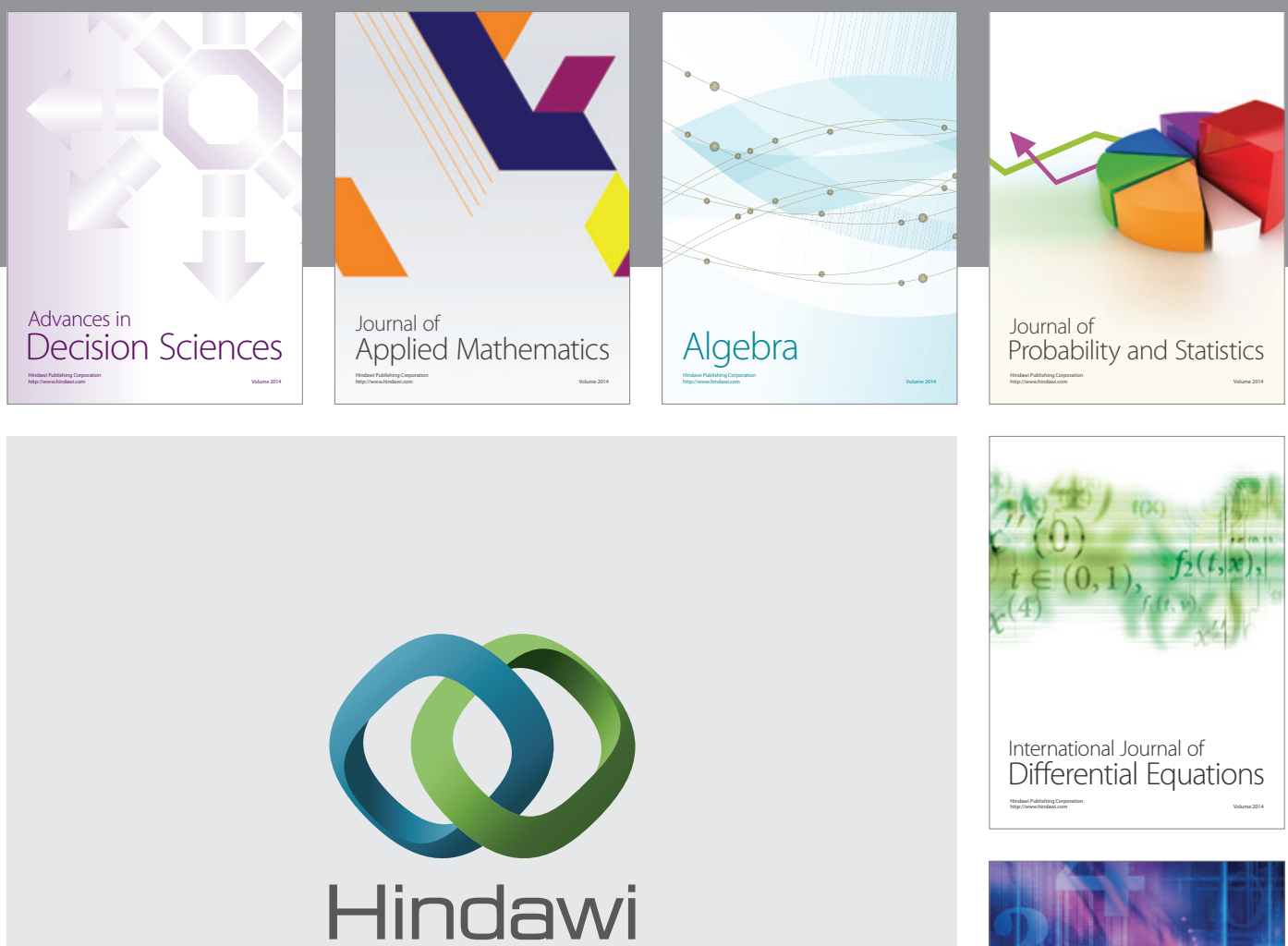

Submit your manuscripts at http://www.hindawi.com
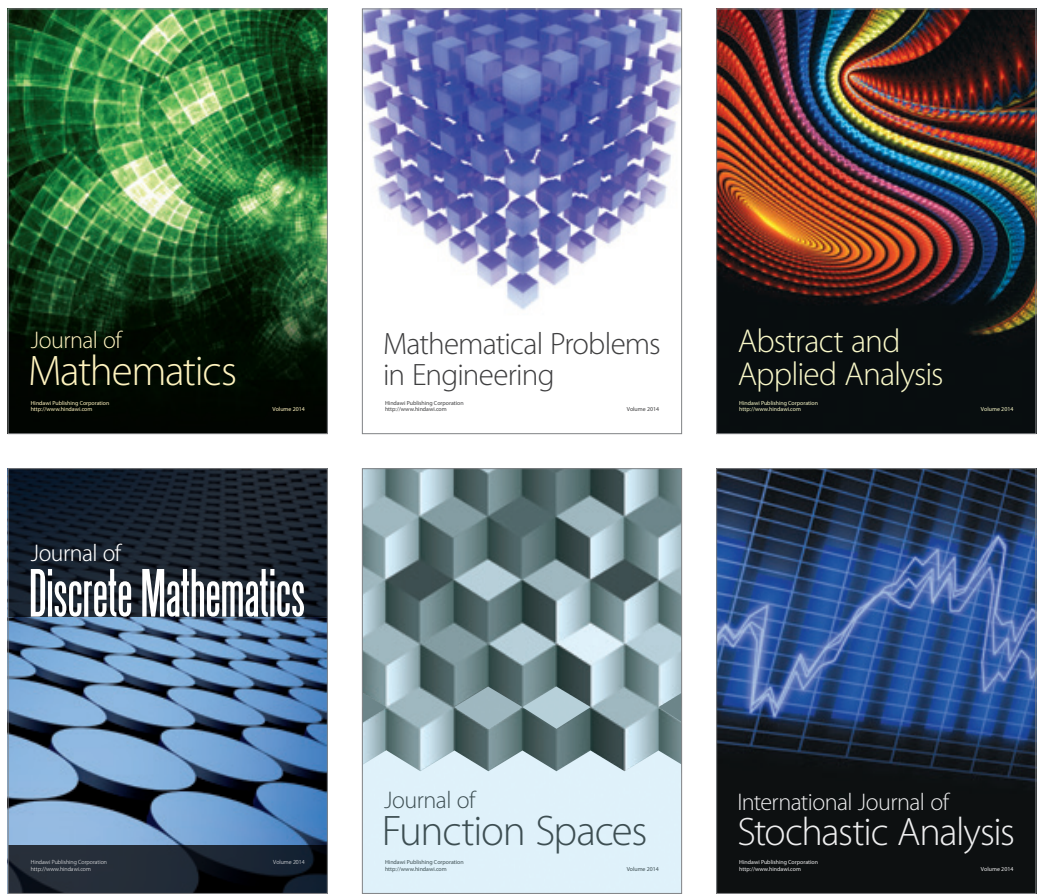

Journal of

Function Spaces

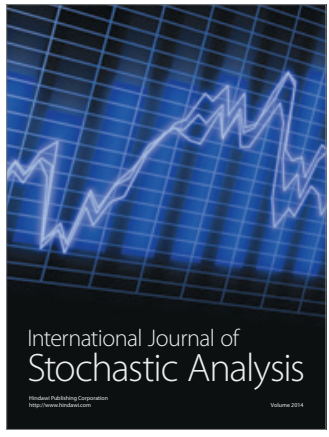

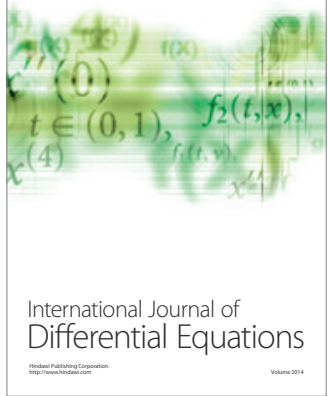
